\title{
Asymptotic localization in the Bose-Hubbard model
}

Alex Bols, and Wojciech De Roeck

Citation: Journal of Mathematical Physics 59, 021901 (2018); doi: 10.1063/1.5022757

View online: https://doi.org/10.1063/1.5022757

View Table of Contents: http://aip.scitation.org/toc/jmp/59/2

Published by the American Institute of Physics

\section{Articles you may be interested in}

Blowup criterion and persistent decay for a modified Camassa-Holm system Journal of Mathematical Physics 59, 021501 (2018); 10.1063/1.5006402

Uniform analytic approximation of Wigner rotation matrices Journal of Mathematical Physics 59, 022102 (2018); 10.1063/1.5012583

Point spectrum of the Floquet Hamiltonian for Klein-Gordon equation under quasi-periodic perturbations

Journal of Mathematical Physics 59, 021503 (2018); 10.1063/1.5023251

Inverse scattering transform for the nonlocal nonlinear Schrödinger equation with nonzero boundary conditions

Journal of Mathematical Physics 59, 011501 (2018); 10.1063/1.5018294

Conormal distributions in the Shubin calculus of pseudodifferential operators Journal of Mathematical Physics 59, 021502 (2018); 10.1063/1.5022778

Scaled lattice fermion fields, stability bounds, and regularity Journal of Mathematical Physics 59, 022301 (2018); 10.1063/1.5022960

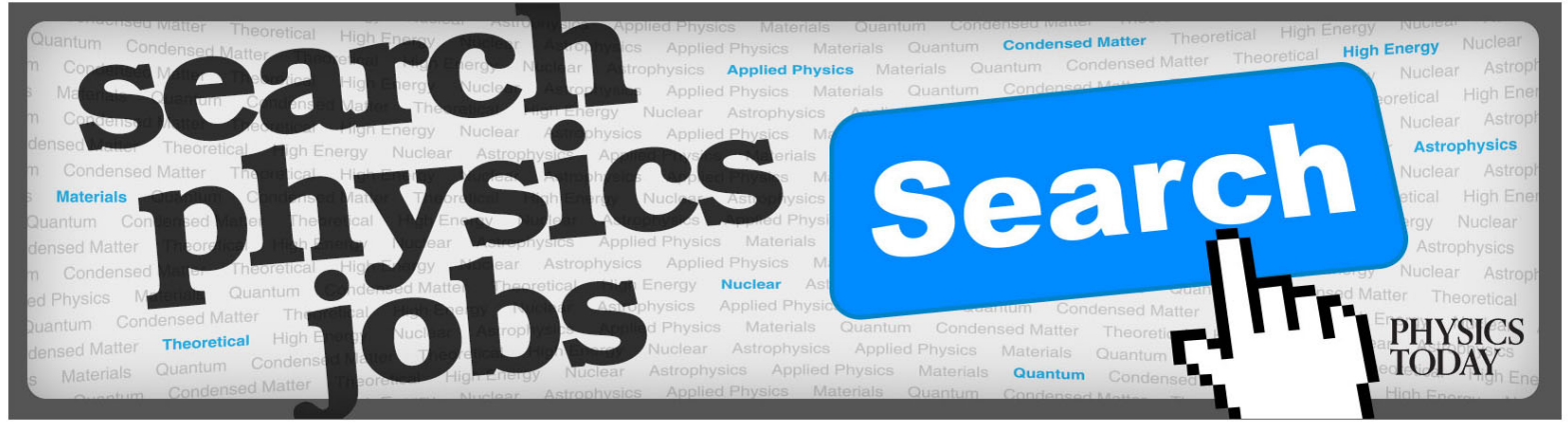




\title{
Asymptotic localization in the Bose-Hubbard model
}

\author{
Alex Bols ${ }^{\mathrm{a})}$ and Wojciech De Roeck ${ }^{\mathrm{b})}$ \\ Instituut voor Theoretische Fysica, K.U.Leuven Celestijnenlaan 200, D 3001 Leuven, Belgium
}

(Received 20 February 2017; accepted 16 January 2018; published online 1 February 2018)

\begin{abstract}
We consider the Bose-Hubbard model. Our focus is on many-body localization, which was described by many authors in such models, even in the absence of disorder. Since our work is rigorous, and since we believe that the localization in this type of models is not strictly valid in the infinite-time limit, we necessarily restrict our study to "asymptotic localization" also known as "quasi-localization:" We prove that transport and thermalization are small beyond perturbation theory in the limit of large particle density. Our theorem takes the form of a many-body Nekhoroshev estimate. An interesting and new aspect of this model is the following: The localization cannot be inferred from a lack of hybridization between zero-hopping eigenstates. Naively speaking, all these eigenstates appear resonant and one has to move to a dressed basis to see the absence of resonances that are responsible for (quasi-)localization. Published by AIP Publishing. https://doi.org/10.1063/1.5022757
\end{abstract}

\section{INTRODUCTION}

\section{A. Many-body localization and asymptotic localization}

Many-body localization (MBL) is a tantalizing phenomenon, which can be considered a breakdown of basic thermodynamics in strongly disordered quantum spin systems. . $^{2} 12,22,24,25,28,29,33,34,45,47$ According to us, the most powerful and useful definition is via the existence of a complete set of quasi-local conserved quantities (also known as LIOMs, "local integrals of motion"); see Refs. 38, 19, and 17. This definition stresses that MBL systems are robustly integrable. A lot of interesting characteristics, like the absence of transport and thermalization, area-law of entanglement, and logarithmic spreading of entanglement, can be deduced from this definition. All of this is discussed at length in the literature; see, e.g., Refs. 11, 20, and 27.

Several authors have proposed that MBL should occur as well in systems without quenched disorder, in particular in translation-invariant systems, see Refs. 5, 6, 8, 16, 21, 36, 35, 13, 46, 31, 14, 23 , and 40 , like the one studied in this paper. Whereas this issue is not settled yet, and in particular, we believe that this claim is false when taken literally ${ }^{7,9}$ (see also the numerics in Refs. 30, 43, and 4), it is clear that a lot of localization phenomenology is present in such systems. To put it succinctly, they behave for a very long time as if they were localized, even if their eigenvectors might eventually be ergodic (i.e., satisfy Eigenstate Thermalization Hypothesis (ETH), see Refs. 10 and 41). In earlier work, we dubbed this property "asymptotic localization" (often one encounters also the term "quasiMBL") stressing the fact that transport and thermalization in such models are non-perturbative effects (weaker than any order in perturbation theory) and hence they are visible only on very long time scales.

There has already been rigorous work establishing this localization phenomenology. ${ }^{6,8,18}$ It should be noted that, unlike for Anderson localization, rigorous results on MBL are scarce, with the notable exception of Ref. 19; see however also Refs. 42, 37, 1, and 26. The present paper establishes asymptotic MBL for the Bose-Hubbard model, which is a natural model due to the interest in cold atom systems. However, the main motivation for this work is that the (asymptotic) localization in

\footnotetext{
a)E-mail: alexander.bols@kuleuven.be

b) E-mail: wojciech.deroeck@kuleuven.be
} 
the Bose-Hubbard model is far more subtle than in most of the translation-invariant systems referred to above. This is explained next.

\section{B. The "quantum localized" versus "classical localized" regime}

In this section, for the sake of focus, we write "localization" without distinguishing between genuine localization and asymptotic localization, but simply referring to "localization phenomena." We also restrict to chains (dimension one). Let us start with a general bosonic chain Hamiltonian of the type

$$
H=\sum_{x} U N_{x}^{q}+J\left(a_{x} a_{x+1}^{*}+a_{x+1} a_{x}^{*}\right)
$$

with $\left[a_{x}, a_{x^{\prime}}^{*}\right]=\delta_{x, x^{\prime}}$ (bosonic fields) and $N_{x}=a_{x}^{*} a_{x}$. For $q=1$, this Hamiltonian describes noninteracting bosons, whereas for $q=2$, we recover the Bose-Hubbard model studied in this paper. The main idea behind localization in this model applies at large mean density $\left\langle N_{x}\right\rangle$ : Consider the energy difference $\Delta E$ resulting from one boson hopping from $x$ to $x+1$,

$$
\Delta E=U\left(\left(N_{x+1}+1\right)^{q}+\left(N_{x}-1\right)^{q}\right)-U\left(N_{x+1}^{q}+N_{x}^{q}\right) \approx U q\left(N_{x+1}^{q-1}-N_{x}^{q-1}\right) .
$$

Since, in most configurations at large density, $N_{x+1}-N_{x}$ is typically large as well, we see that $\Delta E$ is typically increasing with the mean density as soon as $q>1$. The energy difference $\Delta E$ should of course be compared with a typical matrix element of the hopping. The latter is not just of order $J$ because the operators $a_{x}, a_{x}^{*}$ grow with density as well, as $\sqrt{N_{x}}$, and hence the matrix element for the one-boson hop goes roughly as $g \sqrt{N_{x} N_{x+1}}$. So

$$
\frac{\text { matrix element }}{\Delta E} \approx(J / U)\left\langle N_{x}\right\rangle^{2-q},
$$

which suggests that localization occurs for $q>2$ and the Bose-Hubbard model $q=2$ is the critical case, where localization could still be expected for $(J / U) \ll 1$.

However, what the above reasoning shows is simply that there is no localization in the $N_{x}$ basis for $q \leq 2$. That does not exclude localization in some other basis. This type of pitfall is always an issue with heuristic approaches to localization: one argues for localization/delocalization by convergence/divergence of a certain locator expansion, but that is necessarily tied to a choice of basis, which might not be the correct one. A discussion of examples where this happens can be found, e.g., in Ref. 9, the most striking example is the "quantum percolation problem," see Refs. 39 and 44, though one might also consider one-particle localization in the continuum as an instance of this. The above model for $1<q \leq 2$ furnishes in fact a new example of this kind, i.e., a case of "non-obvious" localization.

Indeed, let us discuss at least two indications why the no-localization conclusion for $q \leq 2$ is suspicious:

The non-interacting case $q=1$ : At $q=1$, one has non-interacting bosons. It is then well-known that if $U=U_{x}$ is a random field, then the one-boson problem is exponentially localized. If we secondquantize, then obviously the localization persists in an appropriate sense. It is instructive to look at an extreme toy model where the lattice consists of two points $x=1,2$ and we have two eigenmodes $\phi_{1,2} \in \mathbb{C}^{2}$ strongly localized in the sense that

$$
\left|\phi_{1}(2)\right|^{2}=\left|\phi_{2}(1)\right|^{2}=\epsilon \ll 1 .
$$

The second-quantized, localized base states are $\left|n_{\phi_{1}}, n_{\phi_{2}}\right\rangle \equiv\left(a_{\phi_{1}}^{*}\right)^{n_{\phi_{1}}}\left(a_{\phi_{2}}^{*}\right)^{n_{\phi_{2}}} \Omega$, where $a_{\phi_{i}}^{*}$ $=\sum_{x} \phi_{i}(x) a_{x}^{*}$ are the creation operators of the modes $\phi_{i}$ and $\Omega$ is the vacuum state (no bosons). The states are rather delocalized in the basis of "bare" number states $\left|n_{1}, n_{2}\right\rangle \equiv\left(a_{1}^{*}\right)^{n_{1}}\left(a_{2}^{*}\right)^{n_{2}} \Omega$. They are typically spread out over order of $\sqrt{n}$ states, when $n \approx n_{1} \approx n_{2}$. This means that the hybridization between zero-hopping eigenstates, as predicted from (2) indeed happens, but it does not tell the full story, namely, that there is localization in some other basis.

Semi-classical limit The classical problem resembling (1), namely, the nonlinear Schrödinger chain,

$$
H\left(\psi_{x}\right)=\sum_{x} U\left|\psi_{x}\right|^{2 q}+J\left(\psi_{x} \bar{\psi}_{x+1}+\bar{\psi}_{x} \psi_{x+1}\right)
$$


shows "asymptotic localization" all the way down to $q>1$. This was proved in Ref. 6 for $q=2$ but the argument remains valid for all $q>1$; see Ref. 6 for the relevant heuristics. The model (3) can indeed be viewed as a semi-classical limit of (1) because at large density, the commutator $\left[a_{x}, a_{x}^{*}\right]=1$ is small with respect to the typical values of these operators. We do not develop this theme, started in Ref. 15; see, e.g., Refs. 3 and 15 for references.

These two observations suggest that the localization can be exhibited in a basis that is reminiscent of coherent states (cf. the semi-classical limit alluded to above), in particular different from the basis in which the $N_{x}$ are diagonal. This is indeed what happens. For the sake of simplicity, the present paper deals with the case $q=2$ only, (Bose-Hubbard model) and so we do not focus on this interesting dichotomy between number basis and coherent-like states. Indeed, at the critical case $q=2$, it is the ratio $J / U$ that tunes the hybridization between zero-hopping states. However, the details of the "localization basis" can be read off from the generators of the unitary transformations that approximatively diagonalize the Hamiltonian. The fact that there is hybridization between $N_{x}$-eigenstates then corresponds simply to the statement that, even locally, these generators have large norm when $J / U \gg 1$; see the discussion around Eq. (75) at the end of Sec. IV. In fact, in line with the above remarks, the proofs in this paper are quite similar to those in Ref. 8.

\section{Many-body Nekhoroshev estimates}

Nekhoroshev estimates ${ }^{32}$ in classical mechanics express the fact that dynamics is very slow, also away from KAM tori. In this light, our work can be seen as providing a many-body version of these concepts, though there is no exact analog: Typical Nekhoroshev estimates express that action variables remain close to their original values for long times. We do not prove nor do we believe this to be true in our system. Instead we focus on the energy content $H_{I}$ of intervals $I$, and we show, see Theorem 2.2, that this remains close to its typical value, where the notion of closeness resides in the fact that the difference $H_{I}(t)-H_{I}(0)$ is independent of the length $|I|$. Moreover, this estimate does not apply deterministically but only typically with respect to the Gibbs state. Such a restriction is clearly unavoidable in view of the fact that the localization phenomena depend on high density.

\section{MODEL AND RESULTS}

We define our model with more care for details and we state the results.

\section{A. Model}

Let $N \geq 1$ be an odd integer and let $\mathbb{Z}_{N}=\{-(N-1) / 2, \ldots,(N-1) / 2\}$. We define the Hilbert space

$$
\mathcal{H}_{N}:=\otimes_{x \in \mathbb{Z}_{N}} l^{2}(\mathbb{N}) \sim l^{2}\left(\mathbb{N}^{\mathbb{Z}_{N}}\right),
$$

i.e., at each site labeled by $x \in \mathbb{Z}_{N}$ there is an infinite-dimensional spin-space. For an operator $\hat{O}$ acting on $\mathcal{H}_{N}$, we denote by $s(\hat{O})$ the minimal set $A \subset \mathbb{Z}_{N}$ such that $\hat{O}=\hat{O}_{A} \otimes \mathbb{1}_{\mathbb{Z}_{N} \backslash A}$ for some $\hat{O}_{A}$ acting on $\mathcal{H}_{A}$, and $\mathbb{1}_{A^{\prime}}$ the identity on $\mathcal{H}_{A^{\prime}}$ for any $A^{\prime} \subset \mathbb{Z}_{N}$. We do not distinguish between $\hat{O}_{A}$ and $\hat{O}$, and we will denote both by the same symbol.

Let $\hat{a}, \hat{a}^{*}$ be the usual bosonic annihilation/creation operators on (a dense domain of) $l^{2}(\mathbb{N})$,

$$
(\hat{a} f)(n)=\sqrt{n+1} f(n+1), \quad\left(\hat{a}^{*} f\right)(n+1)=\sqrt{n+1} f(n), \quad \text { for } n \in \mathbb{N} .
$$

We write $\hat{a}_{x}, \hat{a}_{x}^{*}$ for the annihilation/creation operators acting on site $x$. We also define the number operators and the total particle operator

$$
\hat{N}_{x}:=\hat{a}_{x}^{*} \hat{a}_{x}, \quad \hat{N}:=\sum_{x \in \mathbb{Z}_{N}} \hat{N}_{x}
$$

The vectors diagonalizing the operators $\hat{N}_{x}$ play a distinguished role in our analysis. For a finite set $A$, we define the phase space $\Omega_{A}:=\mathbb{N}^{A}$ with elements

$$
\eta=(\eta(x))_{x \in A}, \quad \eta(x) \in \mathbb{N}
$$


such that $\mathcal{H}_{A} \sim l^{2}\left(\Omega_{A}\right)$ and we often use $\eta$ as a label for the function $\delta_{\eta}$, i.e., $\delta_{\eta}\left(\eta^{\prime}\right)=\delta_{\eta, \eta^{\prime}}$ for $\eta$, $\eta^{\prime} \in \Omega_{A}$. We will also write $\Omega_{N}:=\Omega_{\mathbb{Z}_{N}}$.

For each odd integer $N$, we consider the Bose-Hubbard Hamiltonian on $\mathcal{H}_{N}$ with a chemical potential $\mu$ and free boundary conditions,

$$
\hat{H}=\hat{D}+g \hat{V}=\sum_{x \in \mathbb{Z}_{N}} \hat{H}_{x}=\sum_{x \in \mathbb{Z}_{N}}\left(\hat{D}_{x}+g \hat{V}_{x}\right)
$$

with $g \in \mathbb{R}$ being a fixed coupling constant and

$$
\begin{array}{cl}
\hat{D}_{x}=\hat{N}_{x}^{2} & \text { for all } x \in \mathbb{Z}_{N}, \\
\hat{V}_{x}=\hat{a}_{x}^{*} \hat{a}_{x+1}+\hat{a}_{x} \hat{a}_{x+1}^{*} & \text { for } x \in \mathbb{Z}_{N} \backslash\{(N-1) / 2\} \quad \text { and } \hat{V}_{(N-1) / 2}=0 .
\end{array}
$$

The dimensionless coupling constant $g$ plays the role of the ratio $J / U$ mentioned in the Introduction. By the Kato-Rellich theorem, this Hamiltonian is indeed essentially self-adjoint on the dense domain spanned by the vectors $\eta$.

\section{B. States, correlations, and time evolution}

We first introduce a framework to deal with the unbounded operators that will appear in our analysis. Let $\mathcal{A}$ be the ${ }^{*}$-algebra finitely generated by the set

$$
\left\{\hat{a}_{x}, \hat{a}_{x}^{\dagger}, f\left(\hat{N}_{x}\right) \mid f \text { bounded, } x \in \mathbb{Z}_{N}\right\} \text {. }
$$

An important operation on this algebra is the commutator, which we write as

$$
\operatorname{ad}_{\hat{A}_{1}}\left(\hat{A}_{2}\right)=-\operatorname{ad}_{\hat{A}_{2}}\left(\hat{A}_{1}\right)=\left[\hat{A}_{1}, \hat{A}_{2}\right] \text {. }
$$

Next, we define a state $\omega_{\mu}$ (a positive, normalized functional) on $\mathcal{A}$ by

$$
\omega_{\mu}(\hat{A}):=\frac{\operatorname{Tre}^{-\mu \hat{N}} \hat{A}}{\operatorname{Tre}^{-\mu \hat{N}}}, \quad \hat{A} \in \mathcal{A}, \mu>0 .
$$

This corresponds to the grand canonical ensemble at infinite temperature and chemical potential ${ }^{48}-\mu$, so that the mean particle density is finite. As was explained in the Introduction, we expect localization phenomena in the regime of high occupation numbers. We will realise this regime by considering the state $\omega_{\mu}$ at $\mu \ll 1$. Some of our arguments deal with the time evolution generated by the Hamiltonian $H$ defined above. As the latter is self-adjoint, $\mathrm{e}^{-\mathrm{i} t H}, t \in \mathbb{R}$ is a group of unitary operators. However, this time evolution does not leave the algebra $\mathcal{A}$ invariant, and hence we sometimes need a bigger space that we call $\mathcal{B}$. $\mathcal{B}$ is defined as the closure of $\mathcal{A}$ with respect to the weighted Hilbert-Schmidt norm

$$
\|\hat{A}\|_{\omega_{\mu}}:=\left(\omega_{\mu}\left(\hat{A}^{*} \hat{A}\right)\right)^{1 / 2} .
$$

Of course, $\mathcal{B}$ is no longer an algebra, but it inherits the *-involution $A \mapsto A^{*}$ and it is a Hilbert space with inner product $\langle\hat{A}, \hat{B}\rangle=\omega_{\mu}\left(\hat{A}^{*} \hat{B}\right)$. We then check

Lemma 2.1. For any $\hat{B} \in \mathcal{B}$, the operator $\hat{B}(t)=U_{t} \hat{B} U_{t}^{*}$ is well defined as an element of $\mathcal{B}$. The evolution preserves the norm $\omega_{\mu}$, i.e., $\omega_{\mu}\left(\hat{B}(t)^{*} \hat{B}(t)\right)=\omega_{\mu}\left(\hat{B}^{*} \hat{B}\right)$ (it is therefore a unitary group on $\mathcal{B}$, but we do not need this point of view) and, for any $\hat{A} \in \mathcal{A} \subset \mathcal{B}$, we have the following identity in $\mathcal{B}$ :

$$
\hat{A}\left(t_{1}\right)-\hat{A}\left(t_{0}\right)=\mathrm{i} \int_{t_{0}}^{t_{1}} \mathrm{~d} s\left(\operatorname{ad}_{\hat{H}} \hat{A}\right)(s) .
$$

We omit the proof. It is a straightforward application of particle-conservation $[\hat{H}, \hat{N}]=0$ and the fact that $\omega_{\mu}$ contains the exponentially decaying factor $\mathrm{e}^{-\mu \hat{N}}$, taming the polynomial growth of any element in $\mathcal{A}$.

\section{Results}

First we state a result expressing the invariance of the energy content of finite sub-volumes for long times (again polynomial in $\mu^{-1}$ ). This result is reminiscent of Nekhoroshev estimates for 
classical systems with a finite number of degrees of freedom. Let $I=\left\{a_{1}, a_{1}+1, \ldots, a_{2}\right\} \subset \mathbb{Z}_{N}$ be a discrete interval and let $\hat{H}_{I}=\sum_{x \in I} \hat{H}_{x}$. Note that $\hat{H}_{I}=\hat{H}_{I}^{*} \in \mathcal{A}$, and hence $H_{I}(t)$ is well defined. Then

Theorem 2.2. For any $n \geq 1$, there is a number $C_{n}<+\infty$ which is independent of the chain length $N$, such that for any interval I as above we have

$$
\omega_{\mu}\left(\left(\hat{H}_{I}(t)-\hat{H}_{I}(0)\right)^{2}\right) \leq C_{n} \mu^{-4} \quad \text { for any } \quad 0 \leq t \leq \mu^{-n}
$$

if $\mu$ is sufficiently small.

For a thermalizing system, one expects that, during the process of equilibration, the energy content of the interval $I$ changes with time at a rate roughly set by the thermal diffusivity. Once equilibrium is reached after some characteristic time $\tau_{e q}$, the energy in the interval $I$ fluctuates around its equilibrium value, at which point the left-hand side of (14) is expected to scale with the length $|I|$ of the interval. What Theorem 2.2 shows is that the persistent flow of energy into or out of the region $I$ required to equilibrate the system is absent for times that are polynomially long in $\mu^{-1}$. Indeed, we can take the interval $I$ so large that the expected difference of the energy content of the sub-volume $I$ from its equilibrium value is much larger than the bound $\mu^{-4}$ in the right-hand side of (2.2). For an equilibrating system, we would then expect that at some point in time the bound is surpassed because there should be a persistent energy current until the equilibrium energy content is reached, but the theorem shows that these persistent currents are so small that the bound is not passed at times that are polynomially long in $\mu^{-1}$. A fortiori, this shows that the timespan $\tau_{e q}$ needed for the system to reach equilibrium goes to infinity faster than any power of $\mu^{-1}$.

Next, we consider in more detail the occurrence of persistent energy currents at long but finite times. For this purpose, we first introduce some definitions. We define the energy current through the bond $(x, x+1)$ for $x \in \mathbb{Z}_{N}$ by

$$
\hat{J}_{x}:=\mathrm{i} \operatorname{ad}_{\hat{H}} \hat{H}_{>x}, \quad \text { where } \quad \hat{H}_{>x}:=\sum_{y>x} \hat{H}_{y},
$$

i.e., $\hat{J}_{x}$ is the observable corresponding to the rate of change of the energy contained in the part of the chain to the right of the site $x$, which is indeed the energy current across the bond $(x, x+1)$.

The theorem above is a direct consequence of the following abstract result expressing that, to all orders in perturbation in $\mu$, no persistent energy currents can be produced by the dynamics. Only local oscillations of the energy density can be seen at any finite order in $\mu$.

Theorem 2.3. For any integer $n \geq 1$, there is a $\mu_{0}>0$, depending on $n$ but not on the system size $N$, such that for all $\mu \in\left(0, \mu_{0}\right)$ and any site a we can find a number $C_{n}$, independent of $a$ and $N$, and two operators $\hat{U}_{a}=\hat{U}_{a}^{*}$ and $\hat{G}_{a}=\hat{G}_{a}^{*}$ in $\mathcal{A}$ satisfying the following properties:

1. They have vanishing thermal expectation values

$$
\omega_{\mu}\left(\hat{U}_{a}\right)=\omega_{\mu}\left(\hat{G}_{a}\right)=0 .
$$

2. They satisfy the bounds

$$
\omega_{\mu}\left(\hat{U}_{a}^{2}\right) \leq C_{n} \mu^{-4}, \quad \omega_{\mu}\left(\hat{G}_{a}^{2}\right) \leq C_{n} .
$$

3. They are local in the sense that they depend only on sites labeled by $z \in \mathbb{Z}_{N}$ with $|z-a| \leq C_{n}$.

The current across the bond $(a, a+1)$ can be decomposed in terms of these operators as

$$
\hat{J}_{a}=\operatorname{i~ad}_{\hat{H}} \hat{U}_{a}+\mu^{n+1} \hat{G}_{a} .
$$

The proof of this theorem occupies the largest part of this work.

Let us see how it suggests the slowness of transport, even beyond Theorem 2.2, in particular out of equilibrium.

The time-integrated current through the bond $(a, a+1)$ is, by integrating the identity of Theorem 2.3, given by

$$
\int_{0}^{t} \mathrm{~d} s \hat{J}_{a}(s)=\hat{U}_{a}(t)-\hat{U}_{a}(0)+\mu^{n+1} \int_{0}^{t} \mathrm{~d} s \hat{G}_{a}(s)
$$


This seems to be small regardless of the initial state of the system, the point being that the first term on the right-hand side is a temporal boundary term. However, to make this smallness into a mathematical statement, we have to deal with the fact that $U_{a}$ and $G_{a}$ are unbounded operators that can only be controlled on typical states, i.e. states of not too high occupation number. Even if we were to assume that the initial state at time 0 has moderate occupations around $a$ (which suffices because the operators $U_{a}, G_{a}$ are local), then we cannot mathematically guarantee that this remains so up to time $t$. However, this sounds very much like a technical problem and it seems reasonable to conclude that

$$
\left|\omega_{\text {non. eq }}\left(\int_{0}^{t} \mathrm{~d} s \hat{J}_{a}(s)\right)\right| \leq C_{n} \mu^{n+1} t
$$

for almost any reasonable initial state $\omega_{\text {non. eq }}$, in particular a non-equilibrium steady state connected to heat reservoirs at different high temperatures (so that the particle density remains high).

Finally, we note that we formulated our results for energy currents and fluctuations, even though there is another conserved quantity, namely, particle number $\sum_{x} \hat{N}_{x}$. Indeed, analogous theorems can also be formulated for particle number fluctuations and currents. Also, the restriction to infinite temperature and finite $\mu$ is for simplicity, and we could also have taken finite but large temperature. The only important thing is that the average density remains high $\left\langle N_{x}\right\rangle_{\mu, \beta} \gg 1$.

\section{OUTLINE OF THE PROOF}

The proof is inspired by Ref. 8 which in turn picks up on the ideas introduced in Ref. 19.

The first step in the proof is the construction of a change of basis $\hat{\Omega}$ that almost diagonalizes the Hamiltonian $\hat{H}$. We want the unitary operator $\hat{\Omega}$ to be quasi-local in the sense that it is generated by some anti-Hermitian matrix $\hat{K}$ which can be written as a sum of local terms. If we succeed in fully diagonalizing the Hamiltonian in this way, then the transformed Hamiltonian $\hat{\Omega}^{\dagger} \hat{H} \hat{\Omega}$ still has a local structure. Moreover, since it is now also diagonal, its eigenstates $\psi_{x}$ are all localized around some site $x$ in the sense that the decomposition of $\psi_{x}$ in the basis of perfectly localized states $\delta_{y}$ has negligible contributions from localized states that are far away from $x$. It would follow that the Hamiltonian $\hat{H}$ cannot transport energy over a distance greater than that by which the eigenstates $\psi_{x}$ are spread out around the site $x$, i.e., the conductivity would be zero.

Let us try to construct such a change of basis. Note first that the thermal expectation values of the field operators go as $\omega_{\mu}\left(\hat{a}_{x}^{\dagger}\right), \omega_{\mu}\left(\hat{a}_{x}\right) \sim \mu^{-1 / 2}$. Hence $\omega_{\mu}\left(\hat{N}_{x}\right) \sim \mu^{-1}, \omega_{\mu}\left(\hat{H}_{0}\right) \sim \mu^{-2}$, and $\omega_{\mu}(\hat{V}) \sim \mu^{-1}$. Therefore, if $\mu$ is small, in a typical state we have $\hat{H}_{0} \gg \hat{V}$ and we can apply perturbation theory for such states. Now, we want to transform away the interaction terms $\hat{V}=\sum_{x} \hat{a}_{x}^{\dagger} \hat{a}_{x+1}+$ h.c. through the change of basis $\hat{\Omega}=\mathrm{e}^{-\hat{K}}$ and therefore assume that $\hat{K}$ will be of the same order as $\hat{V}$. Let us then expand $\hat{\Omega}^{\dagger} \hat{H} \hat{\Omega}$ to first order in $\hat{V}$ and $\hat{K}$,

$$
\hat{\Omega}^{\dagger} \hat{H} \hat{\Omega}=\mathrm{e}^{\hat{K}}\left(\hat{H}_{0}+\hat{V}\right) \mathrm{e}^{-\hat{K}}=\hat{H}_{0}+\hat{V}+\left[\hat{K}, \hat{H}_{0}\right]+\text { higher order terms. }
$$

We want $\hat{K}$ to solve the equation

$$
\hat{V}=\left[\hat{H}_{0}, \hat{K}\right] .
$$

Let us write $\hat{V}=\sum_{\rho} \hat{V}_{\rho}$, where $\hat{V}_{\rho}$ is a hopping term whose only non-vanishing matrix elements are $\left\langle\eta+\rho\left|\hat{V}_{\rho}\right| \eta\right\rangle$. Then the equation is solved by $\hat{K}=\sum_{\rho} \hat{K}_{\rho}$ with

$$
\left\langle\eta^{\prime}, \hat{K}_{\rho} \eta\right\rangle=\frac{\left\langle\eta^{\prime}, \hat{V}_{\rho} \eta\right\rangle}{\hat{H}_{0}\left(\eta^{\prime}\right)-\hat{H}_{0}(\eta)} .
$$

This is all fine as long as the denominator is not too small. Since the only non-vanishing matrix elements of $\hat{K}_{\rho}$ are $\left\langle\eta+\rho\left|\hat{K}_{\rho}\right| \eta\right\rangle$, and at small $\mu$ the expectation value of $\hat{N}_{x}$ is large, we have to consider

$$
\hat{H}_{0}(\eta+\rho)-\hat{H}_{0}(\eta)=|\eta+\rho|^{2}-|\eta|^{2}=2 \eta \cdot \rho+|\rho|^{2} \approx 2 \eta \cdot \rho .
$$

If this quantity is too small, we say that $\rho$ is resonant at $\eta$ and we simply set $\left\langle\eta+\rho\left|\hat{K}_{\rho}\right| \eta\right\rangle=0$. In this way, we get rid of all non-resonant matrix elements $\left\langle\eta+\rho\left|\hat{V}_{\rho}\right| \eta\right\rangle$ to first order. On the other hand, the higher order terms in (16) give new local interaction terms in the transformed Hamiltonian beyond the nearest neighbour interactions of the original Hamiltonian. The long range interactions generated 
in this way are small, and therefore we can restrict our attention to those terms which may induce hopping over some finite range $r$. (In the actual proof, we truncate the expansion of $\hat{\Omega}=\mathrm{e}^{-\hat{K}}$ and we do not generate interaction terms of arbitrary range at all, but this is a technical point.)

We can now iterate this procedure, at each step trying to transform away the next order nonresonant interactions of the Hamiltonian obtained from the previous change of basis.

We end up with a Hamiltonian

$$
\widetilde{H}=\hat{H}_{0}+\sum_{\rho} \widetilde{V}_{\rho}
$$

where

$$
\left\langle\eta+\rho, \widetilde{V}_{\rho} \eta\right\rangle=0 \quad \text { if } \quad|\eta \cdot \rho|>C .
$$

and all hopping vectors $\rho$ are supported on sites lying in some interval of length at most $r$ and $\left|\rho_{x}\right| \leq r$. For each such finite-range hopping $\rho$ in phase space, the condition $|\eta \cdot \rho| \leq C$ defines a thickened hyperplane in phase space where transport is possible. Note that the projection on this hyperplane has small probability with respect to the Gibbs state if $\mu$ is small, so in this sense resonances are rare. Since the transformation to the resonant Hamiltonian is local, it is now sufficient to show that the energy current defined by the resonant Hamiltonian is small. This current is given by

$$
\widetilde{J}_{a}=\mathrm{i}\left[\widetilde{H}, \widetilde{H}_{>a}\right],
$$

where for any $x \in \mathbb{Z}$ we split the resonant Hamiltonian into a left part $\widetilde{H}_{\leq x}$ and a right part $\widetilde{H}_{>x}$ by assigning the local terms in $\widetilde{H}$ either to the left or to the right. The only ambiguity in this splitting is for the terms $\widetilde{V}_{\rho}$ with $\rho$ a resonance that spans the bond $(x, x+1)$; we can arbitrarily assign these terms to the right part of the splitting.

The probability that there is no resonance across the bond $(a, a+1)$, and thus no current across this bond, is small in $\mu$. But it is only polynomially small in $\mu$ and so we need to do better. The idea is to look for a bond $(x, x+1)$ as close to the bond $(a, a+1)$ as possible, and across which no instantaneous current flows. There are of course very resonant states for which the closest bond without resonance is arbitrarily far from the bond $(a, a+1)$ so we will have to impose some limit on how far to look for a resonance free bond. Let us say that if we can find a resonance free bond $(x, x+1)$ with $x \in B(a, n):=\left\{y \in \mathbb{Z}_{N}:|a-y| \leq n\right\}$, then we define $x(\eta)=x$. If there is no resonance free bond $(x, x+1)$ with $x \in B(a, n)$, then we give up and simply put $x(\eta)=a$. Note that this means that there is a resonance across $2 n$ bonds. In the latter scenario, we only bound the current across the bond $(a, a+1)$ by a quantity of order one, but the probability of this occurring goes as $\mu^{n c}$ with $c$ some constant of order one. Since $n$ is arbitrary, this is sufficiently improbable for us to bound the Green-Kubo conductivity by an arbitrary power of $\mu$.

Let us try to put some more flesh on this idea. For each $x \in B(a, n)$, we construct an indicator function $\theta_{x}$ on the phase space which equals one if, in the state $\eta$, the current through the bond $(x, x+1)$ vanishes and equals zero if it does not, i.e., $\theta_{x}(\eta)=1$ if there are no hopping vectors $\rho$ spanning the bond $(x, x+1)$ for which the resonance condition $|\eta \cdot \rho| \leq C$ is satisfied, and $\theta_{x}(\eta)=0$ if there does exist such a hopping vector.

We then define $x(\eta)$ to be $x \in B(a, n)$ such that $\theta_{x}(\eta)=1$ which is closest to $a$ if such an $x$ exists, and $x(\eta)=a$ otherwise. In this way, we obtain a state-dependent splitting of the Hamiltonian,

$$
\widetilde{H}_{L}:=\sum_{\eta} \widetilde{H}_{\leq x(\eta)} \hat{P}_{\eta}, \quad \widetilde{H}_{R}:=\sum_{\eta} \widetilde{H}_{>x(\eta)} \hat{P}_{\eta},
$$

and the current becomes

$$
\widetilde{J}_{a}=\mathrm{i}\left[\widetilde{H}, \widetilde{H}_{>a}\right]=\mathrm{i}\left[\widetilde{H},\left(\widetilde{H}_{>a}-\widetilde{H}_{R}\right)\right]+\mathrm{i}\left[\widetilde{H}, \widetilde{H}_{R}\right] .
$$

The first term is the flow of the local operator $\widetilde{H}_{>a}-\widetilde{H}_{R}$. This operator is sparse in the sense that for most $\eta$ the operators $\widetilde{H}_{>a}$ and $\widetilde{H}_{R}$ are equal, so when we integrate the current over long times we get a small contribution that does not scale with time. The first term is therefore harmless. To analyse the second term, we define projectors

$$
\hat{P}_{x}:=\sum_{\eta: x(\eta)=x} \hat{P}_{\eta}
$$


so that

$$
\widetilde{H}_{R}=\sum_{x} \widetilde{H}_{>x} \hat{P}_{x}
$$

We then find

$$
\left[\widetilde{H}, \widetilde{H}_{R}\right]=\sum_{x}\left(\left[\widetilde{H}, \widetilde{H}_{>x}\right] \hat{P}_{x}+\widetilde{H}_{>x}\left[\widetilde{H}, \hat{P}_{x}\right]\right) .
$$

The states that are not annihilated by the projector $\hat{P}_{x}$ have no resonances across the bond $(x, x+1)$, and therefore the commutator $\left[\widetilde{H}, \widetilde{H}_{>x}\right]$ vanishes on those states, so the first term in the summand is under control. The second term in the summand is not a priori small. It would be small however if the projectors $\hat{P}_{x}$ are invariant under the flow, but these projectors are functions of the indicators $\theta_{x}$. Our goal is therefore to construct indicators $\theta_{x}$ that tell us whether there are resonances around the bond $(x, x+1)$ and, at the same time, are (almost) invariant under the flow of $\widetilde{H}$.

To this end, we will investigate the geometry of resonances around the site $x$. Remember that the states at which a given $\rho$ is resonant lie within a thickened hyperplane in the phase space defined by $|\eta \cdot \rho| \leq C$, i.e., the hopping vector $\eta$ is resonant in some neighbourhood of the hyperplane $\pi(\rho):=\{\eta: \eta \cdot \rho=0\}$. States that are multi-resonant, say with hopping vectors $\rho_{1}, \ldots, \rho_{p}$, are to be found in the neighbourhood of $\pi\left(\rho_{1}, \ldots, \rho_{p}\right):=\pi\left(\rho_{1}\right) \cap \cdots \cap \pi\left(\rho_{p}\right)$. We will construct sets $\mathrm{B}\left(\rho_{1}, \ldots, \rho_{p}\right)$ that contain the $p$-fold resonances around $\pi\left(\rho_{1}, \ldots, \rho_{p}\right)$ and are invariant under moves $\eta \rightarrow \eta+\rho$ for any hopping vector $\rho \in \operatorname{span}\left\{\rho_{1}, \ldots, \rho_{p}\right\}$.

Let us denote by $P(\eta, E)$ the orthogonal projection of $\eta$ on the subspace $E$. Let $L$ be a large number; we say that $\eta \in \mathrm{B}\left(\rho_{1}, \ldots, \rho_{p}\right)$ if

$$
\left|\eta-P\left(\eta, \pi\left(\rho_{1}, \ldots, \rho_{p}\right)\right)\right|_{2} \leq C L^{p}
$$

and if, for every linearly independent set of hopping vectors $\rho_{1}^{\prime}, \ldots, \rho_{p^{\prime}}^{\prime} \in \operatorname{span}\left\{\rho_{1}, \ldots, \rho_{p}\right\}$,

$$
\left|P\left(\eta, \pi\left(\rho_{1}^{\prime}, \ldots, \rho_{p^{\prime}}^{\prime}\right)\right)-P\left(\eta, \pi\left(\rho_{1}, \ldots, \rho_{p}\right)\right)\right|_{2} \leq C\left(L^{p}-L^{p^{\prime}}\right) .
$$

The purpose of the first condition is clear, and the set $\mathrm{B}\left(\rho_{1}, \ldots, \rho_{p}\right)$ consists of states $\eta$ in a very broad cylinder around $\pi\left(\rho_{1}, \ldots, \rho_{p}\right)$. In particular, any state that has resonant hopping vectors $\rho_{1}, \ldots, \rho_{p}$ will be contained in the set $\mathrm{B}\left(\rho_{1}, \ldots, \rho_{p}\right)$.

But this cylinder is clearly not invariant under resonant moves by $\rho \in \operatorname{span}\left\{\rho_{1}, \ldots, \rho_{p}\right\}$. Indeed, for any set of hopping vectors $\rho_{1}^{\prime}, \ldots, \rho_{p^{\prime}}^{\prime} \in \operatorname{span}\left\{\rho_{1}, \ldots, \rho_{p}\right\}$, the set $\pi\left(\rho_{1}^{\prime}, \ldots, \rho_{p^{\prime}}^{\prime}\right)$ intersects the boundary of $\mathrm{B}\left(\rho_{1}, \ldots, \rho_{p}\right)$. The surface of the cylinder is curved, so around these intersections there are states that can hop from the inside of the cylinder to the outside or vice versa. The remedy is to flatten the surface of the cylinder in the neighbourhood of the intersection, which is the purpose of the second condition defining the set $\mathrm{B}\left(\rho_{1}, \ldots, \rho_{p}\right)$. This is illustrated in Fig. 1(d) where the resonant flow is indicated by green arrows. It is clear that jumps along the green arrows leave the flattened sphere invariant.

By taking $L$ large enough the flattened parts of the cylinder can be guaranteed to be much longer than the distance of any hopping vector. Furthermore, the flattenings are constructed such that the width of the flattening produced by hopping vectors $\rho_{1}^{\prime}, \ldots, \rho_{p^{\prime}}^{\prime}$ is of order $L^{p^{\prime} / 2}$, i.e., higher order resonances give rise to wider flattenings that cut deeper into the cylinder. To see why this is important, consider the construction of $\mathrm{B}\left(\rho_{1}, \rho_{2}, \rho_{3}\right)$ for $\rho_{1}, \rho_{2}, \rho_{3}$ three independent hopping vectors which is illustrated in Fig. 1. First, as in Fig. 1(a), we peel off thin ring-shaped shells to create the flattenings for each individual hopping vector in $\operatorname{span}\left\{\rho_{1}, \rho_{2}, \rho_{3}\right\}$. This makes the set invariant under all those hoppings except around the places where two or more of such rings intersect as in Fig. 1(b). But this situation is rectified by the flattening corresponding to sets of two hopping vectors in span $\left\{\rho_{1}, \rho_{2}, \rho_{3}\right\}$ which is constructed in Fig. 1(c).

We now construct such a set $\mathrm{B}\left(\rho_{1}, \ldots, \rho_{p}\right)$ for each set of linearly independent hopping vectors $\left\{\rho_{1}, \ldots, \rho_{p}\right\}$ with $p \leq n$ whose support percolates and such that at least one of the hopping vectors has support near to the site $x$ for which we want to construct the invariant indicator $\theta_{x}$. We call such sets of hopping vector clusters around $x$. We then put $\theta_{x}(\eta)=0$ if there is a cluster $\left\{\rho_{1}, \ldots, \rho_{p}\right\}$ around $x$ such that $\eta \in \mathrm{B}\left(\rho_{1}, \ldots, \rho\right)$ and $\theta_{x}(\eta)=1$ otherwise. 


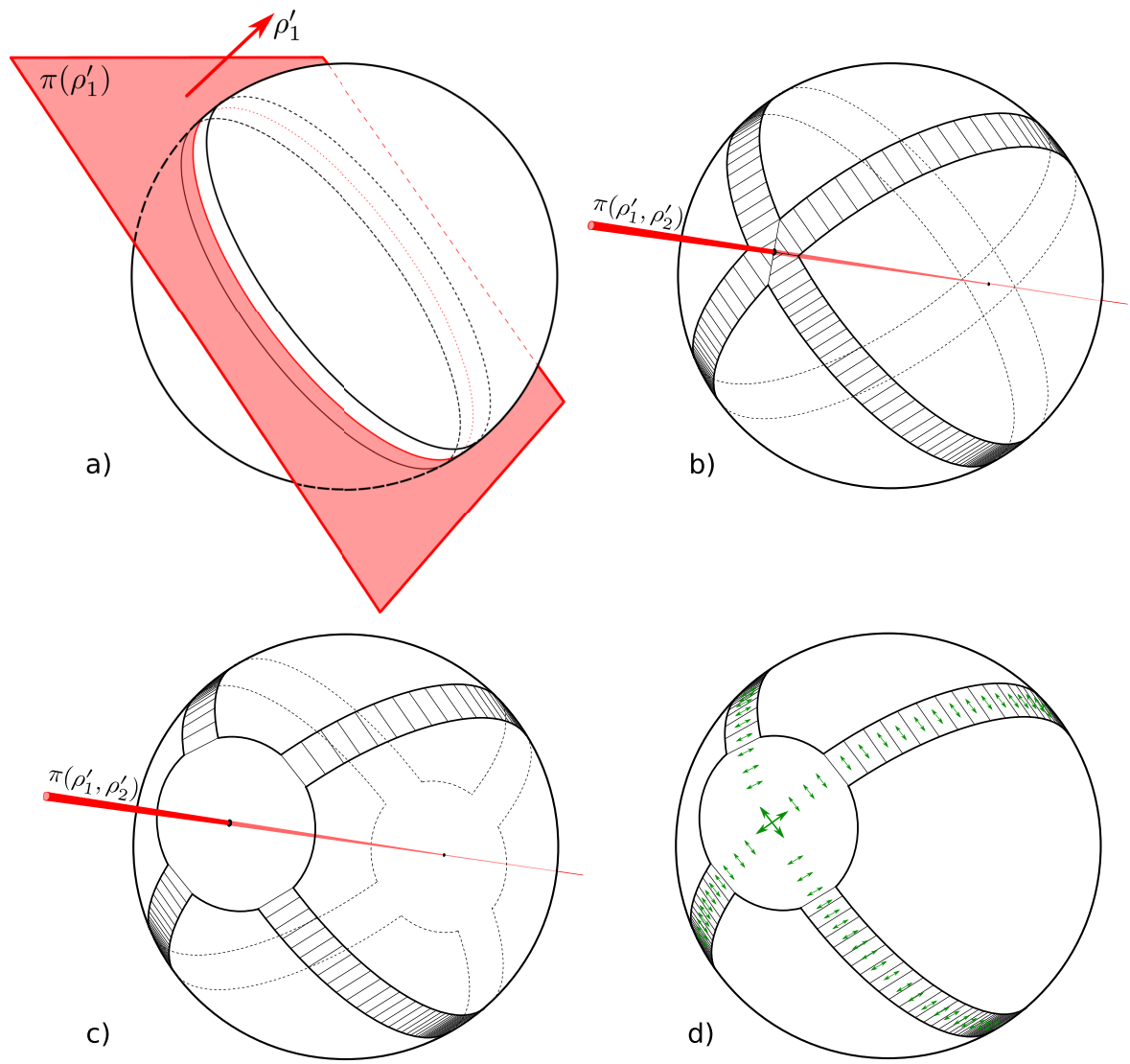

FIG. 1. Construction of $\mathrm{B}\left(\rho_{1}, \rho_{2}, \rho_{3}\right)$. We start with a sphere of radius $C L^{3}$ centred on the multi-resonance $\pi\left(\rho_{1} m \rho_{2}, \rho_{3}\right)$. In (a), we consider a hopping vector $\rho_{1}^{\prime} \in \operatorname{span}\left\{\rho_{1}, \rho_{2}, \rho_{3}\right\}$ and we flatten the sphere where the resonant hyperplane $\pi\left(\rho_{1}^{\prime}\right)$ intersects the sphere. The width of the flattened strip is of order $L$. The result of doing this for one more hopping vector $\rho_{2}^{\prime}$ is shown in (b). The flattenings overlap in the neighbourhood of the multi-resonance $\pi\left(\rho_{1}^{\prime}, \rho_{2}^{\prime}\right)$ and it is easily seen there that the flattened sphere is not invariant under hoppings by $\rho_{1}^{\prime}$ or $\rho_{2}^{\prime}$. This situation is rectified in (c) by an extra flattening around the multi-resonance $\pi\left(\rho_{1}^{\prime}, \rho_{2}^{\prime}\right)$. The radius of this flattened disc is of order $L^{2}$. Finally, the end product is displayed in (d) where we also indicated the resonant hopping vectors near the surface of the set in green.

First of all, if $\rho$ is a hopping vector whose support spans the bond $(x, x+1)$, then $\rho$ by itself is a cluster around $x$. So if $\rho$ is resonant at $\eta$, then $\eta \in \mathrm{B}(\rho)$; hence $\theta_{x}(\eta)=0$. If on the other hand $\eta$ has no resonances around the bond $(x, x+1)$, then $\theta_{x}(\eta)=1$. We see then that $\theta_{x}$ indeed tells us whether or not there is a resonance spanning the bond $(x, x+1)$ as was required. It is also clear that the probability with respect to the Gibbs state of having $\theta_{x}(\eta)=0$ is polynomially small in $\mu$.

We turn now to the invariance properties of the function $\theta_{x}$. Fix $\eta$ and let $\rho$ be resonant at $\eta$. We want to show that $\theta_{x}(\eta)=\theta_{x}(\eta+\rho)$. There are three possible scenarios:

1. There is a cluster $\left\{\rho_{1}, \ldots, \rho_{p}\right\}$ around $x$ such that $\rho \in \operatorname{span}\left\{\rho_{1}, \ldots, \rho_{p}\right\}$ and $\eta \in \mathrm{B}\left(\rho_{1}, \ldots, \rho_{p}\right)$. But the set $\mathrm{B}\left(\rho_{1}, \ldots, \rho_{p}\right)$ was constructed specifically to be invariant under hoppings in span $\left\{\rho_{1}, \ldots, \rho_{p}\right\}$, so $\theta_{x}(\eta)=\theta_{x}(\eta+\rho)=0$.

2. There is a cluster $\left\{\rho_{1}, \ldots, \rho_{p}\right\}$ around $x$ such that $\rho \perp \operatorname{span}\left\{\rho_{1}, \ldots, \rho_{p}\right\}$ and $\eta \in \mathrm{B}\left(\rho_{1}, \ldots, \rho_{p}\right)$. Then the set $\mathrm{B}\left(\rho_{1}, \ldots, \rho_{p}\right)$ is manifestly invariant under translations in the direction of $\rho$ and so we have invariance.

3. If neither scenario 1 nor scenario 2 is the case, then for each cluster $\left\{\rho_{1}, \ldots, \rho_{p}\right\}$ around $x$ such that $\eta \in \mathrm{B}\left(\rho_{1}, \ldots, \rho_{p}\right)$ we have neither $\rho \in \operatorname{span}\left\{\rho_{1}, \ldots, \rho_{p}\right\}$ nor $\rho \perp \operatorname{span}\left\{\rho_{1}, \ldots, \rho_{p}\right\}$. In this case, we must have $p=n$ for if $p<n$ we see that $\rho \cup\left\{\rho_{1}, \ldots, \rho_{p}\right\}$ is also a cluster around $x$, and since $\rho$ is a resonance we have $\eta$ close enough to $\pi\left(\rho, \rho_{1}, \ldots, \rho_{p}\right)$ to conclude that also $\eta \in \mathrm{B}\left(\rho, \rho_{1}, \ldots, \rho_{p}\right)$ which contradicts the assumption. So in this scenario, we have 
$\eta \in \mathrm{B}\left(\rho_{1}, \ldots, \rho_{n}\right)$, i.e., $\eta$ is close to resonance with $n$ linearly independent hopping vectors. The probability of this event is exponentially small in $n$, it goes to zero as some power of $\mu^{n}$.

We conclude that the indicators $\theta_{x}$ are invariant up to a very sparse set. Since the projectors $\hat{P}_{x}$ depend only on a finite number of these indicators, they are also invariant up to a very sparse set and it follows that the commutator $\left[\widetilde{H}, \hat{P}_{x}\right]$ appearing in (27) has a very small expectation value with respect to the Gibbs state at small chemical potential.

Let us take stock of what we have achieved. In (24), we wrote the current in the form

$$
\widetilde{J}_{a}=\mathrm{i}\left[\widetilde{H}, \widetilde{U}_{a}\right]+\widetilde{G}_{a}
$$

with $\widetilde{U}_{a}$ being a local observable whose support is centred on the site $a$ and which has a finite expectation value with respect to the Gibbs state. The operator $\widetilde{G}_{a}$ is also local because it is the difference of two local operators $\widetilde{J}_{a}$ and $\left[\widetilde{H}, \widetilde{U}_{a}\right]$. Furthermore, the Gibbs expectation value of the operator $\widetilde{G}_{a}$ is bounded by $\mu^{c n}$ for some number $c>0$. This is the content of Theorem 2.3 .

\section{REMOVAL OF NON-RESONANT TERMS FROM THE HAMILTONIAN}

In this section, we begin the proof of our results. From this point up to Sec. VII, all equalities between operators are to be understood as identities in the ${ }^{*}$-algebra $\mathcal{A}$ as no topology will be required. In Sec. VII, we do need the topology to define the time evolution of operators and we will therefore move to the Hilbert space $\mathcal{B}$. Throughout, we call operators $\hat{A} \in \mathcal{A}$ symmetric if $\hat{A}=\hat{A}^{*}$ and antisymmetric if $\hat{A}=-\hat{A}^{*}$. We will construct operators in $\mathcal{A}$ which we will call 'Hamiltonians' (often denoted by symbols $h, \widetilde{h}, \ldots$ ), but this simply indicates that they are symmetric elements of $\mathcal{A}$.

\section{A. Preliminary definitions}

Let $\delta \in(0,1)$ be a number. We define reduced annihilation and creation operators by $\hat{\alpha}_{x}:=\delta^{1 / 2} \hat{a}_{x}$ and $\hat{\alpha}_{x}^{*}:=\delta^{1 / 2} \hat{a}_{x}^{*}$. We also define reduced number operators by $\hat{n}_{x}:=\hat{\alpha}_{x}^{*} \hat{\alpha}_{x}=\delta \hat{N}_{x}$.

Suppose $\mu \in(0,1)$ and consider the Hamiltonian

$$
\hat{h}=\hat{d}+\mu \hat{v}=\sum_{x \in \mathbb{Z}_{N}} \hat{h}_{x}=\sum_{x \in \mathbb{Z}_{N}}\left(\hat{d}_{x}+\mu \hat{v}_{x}\right)
$$

with

$$
\begin{aligned}
\hat{d}_{x}=\hat{n}_{x}^{2} & \text { for all } x \in \mathbb{Z}_{N}, \\
\hat{v}_{x}=g\left(\hat{\alpha}_{x}^{*} \hat{\alpha}_{x+1}+\hat{\alpha}_{x} \hat{\alpha}_{x+1}^{*}\right) & \text { for } x \in \mathbb{Z}_{N} \backslash\{(N-1) / 2\}, \text { and } \hat{v}_{(N-1) / 2}=0 .
\end{aligned}
$$

By putting $\delta=\mu$ and multiplying the reduced Hamiltonian $\hat{h}$ by $\mu^{-2}$, we recover the Bose-Hubbard Hamiltonian, but for now we want to think of $\mu$ and $\delta$ as independent variables.

Throughout all this work, we will deal with operators $\hat{f}$ in a subspace $\mathcal{S}$ of the algebra $\mathcal{A}$. An operator $\hat{f}$ belongs to $\mathcal{S}$ if the following conditions are realized for some number $r=r(\hat{f})>0$ :

1. $\hat{f}$ can be written as a sum of local terms,

$$
\hat{f}=\sum_{x} \hat{f}_{x} \quad \text { with } \quad s\left(\hat{f}_{x}\right) \subset \mathrm{B}(x, r),
$$

where $\mathrm{B}(x, r):=\left\{y \in \mathbb{Z}_{N}:|x-y| \leq r\right\}$.

2. $\hat{f}$ has a limited range in the phase space,

$$
\hat{P}_{\eta^{\prime}}, \hat{f} \hat{P}_{\eta}=0 \quad \text { whenever } \quad\left|\eta-\eta^{\prime}\right|_{1}>r .
$$

Also, it follows from spacial locality that $\hat{P}_{\eta^{\prime}} \hat{f} \hat{P}_{\eta}=0$ if $\eta-\eta^{\prime}$ is not supported on a ball of radius $r$.

3. The local terms $\hat{f}_{x}$ can be written in the form

$$
\hat{f}_{x}=\sum_{\substack{\rho \in M_{r} \\ \operatorname{Supp}(\rho) \subset \mathrm{B}(x, r)}} \hat{f}_{x}^{(\rho)}=\sum_{\substack{\rho \in M_{r} \\ \operatorname{Supp}(\rho) \subset \mathrm{B}(x, r)}} \hat{A}_{\rho} \hat{b}_{x, \rho},
$$


where $\operatorname{Supp}(\rho):=\left\{x \in \mathbb{Z}_{N}: \rho_{x} \neq 0\right\}$ is the support of the vector $\rho$, the set $M_{r}:=\left\{\rho \in \mathbb{Z}^{N}\right.$ : $\operatorname{Supp}(\rho) \subset \mathrm{B}(x, r)$ for some $x \in \mathbb{Z}_{N}$ and $\left.\max _{x \in Z_{N}}\left|\rho_{x}\right| \leq r\right\}$ is the set of moves of range $r$, the operators $\hat{A}_{\rho}$ are monomials of reduced annihilation and creation operators whose degree is bounded independently of $\delta$ and such that $\hat{P}_{\eta^{\prime}} \hat{A}_{\rho} \hat{P}_{\eta}=0$ unless $\eta^{\prime}-\eta=\rho$, and the operators $\hat{b}_{x, \rho}$ are bounded and diagonal in the number basis. We also write

$$
\hat{f}^{(\rho)}=\sum_{x} \hat{f}_{x}^{(\rho)}
$$

so that

$$
\hat{f}=\sum_{\rho \in M_{r}} \hat{f}^{(\rho)} .
$$

Note that while the decomposition in local terms may not be unique, this decomposition according to moves is unique.

Let $\xi \in C^{\infty}(\mathbb{R},[0,1])$ be a smooth cut-off function: $\xi(-x)=\xi(x)$ for every $x \in \mathbb{R}, \xi(x)=1$ for every $x \in[-1,1]$, and $\xi(x)=0$ for every $x \notin[-2,2]$. For any $a>0$, we define also $\xi_{a}$ by $\xi_{a}(x)$ $=\xi(x / a)$.

Considering the phase space $\Omega_{N}=\mathbb{N}^{N}$ as a subset of $\mathbb{R}^{N}$, we associate to any function $b: \mathbb{R}^{N}$ $\rightarrow V \subset \mathbb{C}$ a diagonal operator $\hat{b}$ defined by $\hat{b} P_{\eta}=b(\eta) P_{\eta}$ for all $\eta \in \Omega_{N}$. Conversely, any diagonal operator $\hat{b}$ can be considered associated with some function $b$ on $\mathbb{R}^{N}$ and this fact will be used without comment.

For any diagonal operator $\hat{b}$ and any $\rho \in \mathbb{Z}^{N}$, we define the discrete derivative of $\hat{b}$ in the direction $\rho$ as the diagonal operator $\Delta_{\rho} \hat{b}$ given by

$$
\left(\Delta_{\rho} \hat{b}\right) \hat{P}_{\eta}=(b(\eta+\rho)-b(\eta)) \hat{P}_{\eta}
$$

for all $\eta \in \Omega_{N}$.

We associate with the operator $\Delta_{\rho} \hat{b}$ a function

$$
\Delta_{\rho} b: \mathbb{R}^{N} \rightarrow \mathbb{C}: \eta \mapsto b(\eta+\rho)-b(\eta) .
$$

Note that the commutator of a product of annihilation and creation operators with a diagonal operator produces a discrete derivative,

$$
\left[\hat{A}_{\rho}, \hat{b}\right]=\left(\Delta_{\rho} \hat{b}\right) \hat{A}_{\rho} .
$$

We define $E(\eta)=\sum_{x} \eta_{x}^{2}$ corresponding to the on-site energy of the Bose-Hubbard Hamiltonian and associated with this function the diagonal operators $\hat{E}$ and $\Delta_{\rho} \hat{E}$ for any $\rho \in \mathbb{Z}^{N}$ as described above.

Let $1 / 2<\gamma<1$ and define for any $\rho \in \mathbb{Z}^{N}$ the composite function $\zeta_{\rho}: \mathbb{R}^{N} \rightarrow[0,1]: \eta$ $\mapsto \xi_{\delta^{-\gamma}}\left(\Delta_{\rho} E(\eta)\right)$ and the associated diagonal operator $\hat{\zeta}_{\rho}$.

We define an operator $\mathcal{R}$ acting on $\mathcal{S}$ as

$$
\mathcal{R} \hat{f}=\sum_{\rho \in M_{r}} \hat{f}^{(\rho)} \hat{\zeta}_{\rho}
$$

i.e., $\mathcal{R} \hat{f}$ consists of the resonant terms of $\hat{f}$.

Given $\hat{f} \in \mathcal{S}$, the equation

$$
\operatorname{ad}_{\hat{d}} \hat{u}=(\mathrm{Id}-\mathcal{R}) \hat{f}
$$

has a solution in $\mathcal{S}$ given by

$$
\hat{u}=\delta^{-2} \sum_{\rho \in M_{r(f)}} \hat{f}^{(\rho)} \frac{\left(\mathbb{1}-\hat{\zeta}_{\rho}\right)}{\Delta_{\rho} \hat{E}},
$$

where it is understood that the summand on the right-hand side maps $|\eta|$ to zero if $\Delta_{\rho} E(\eta)=0$. We will refer to the operator $\hat{u}$ as the solution to Eq. (41). It is easily checked that if $\hat{f}$ is symmetric, then $\hat{u}$ is antisymmetric. 
Given a vector space $X$, a formal power series in $\mu$ is an expression of the form $Y=\sum_{k \geq 0} \mu^{k} Y^{(k)}$, where $Y^{(k)} \in X$ for every $k \geq 0$. We naturally extend all algebraic operations in $X$ to operations on formal series. Given $l \geq 0$ and given a formal series $Y$, we define the truncation

$$
\mathcal{T}_{l}(Y):=\sum_{k=0}^{l} \mu^{k} Y^{(k)}
$$

If a formal power series $Y$ is such that $Y^{(k)}=0$ for all $k>l$ for some $l \in \mathbb{N}$, we will allow ourselves to identify $Y$ with its truncation $\mathcal{T}_{l}(Y) \in X$.

\section{B. Perturbative diagonalization}

Given $k \geq 1$, let $\pi(k) \subset \mathbb{N}^{k}$ be the collection of $k$-tuples $j=\left(j_{l}\right)_{l=1 \ldots k}$ of non-negative integers satisfying the constraint

$$
\sum_{l=1}^{k} l j_{l}=k
$$

In particular, we have $0 \leq j_{l} \leq k$.

For $k \geq 0$, we recursively define operators $Q^{(k)}, R^{(k)}$, and $S^{(k)}$ on $\mathcal{S}$ as well as operators $\hat{u}^{(k)} \in \mathcal{S}$. Here and below, let us adopt the convention $A^{0}=$ Id for an operator $A$ on $\mathcal{S}$. We first set $Q^{(0)}=R^{(0)}=\mathrm{Id}, S^{(0)}=0$, and $\hat{u}^{(0)}=0$. Next, for $k \geq 1$, we define $\hat{u}^{(k)}$ as the solution to the equation

$$
\operatorname{ad}_{\hat{d}} \hat{u}^{(k)}=(\operatorname{Id}-\mathcal{R})\left(S^{(k-1)} \hat{d}+Q^{(k-1)} \hat{v}\right)
$$

and then set

$$
\begin{aligned}
Q^{(k)} & =\sum_{\substack{j \in \pi(k) \\
j_{1} ! \cdots j_{k} !}} \mathrm{ad}_{\hat{u}^{(k)}}^{j_{k}} \cdots \mathrm{ad}_{\hat{u}^{(1)}}^{j_{1}}, \\
R^{(k)} & =\sum_{\substack{j \in \pi(k) \\
j^{\prime}}} \frac{(-1)^{j_{1}+\cdots+j_{k}}}{j_{1} ! \cdots j_{k} !} \mathrm{ad}_{\hat{u}^{(1)}}^{j_{1}} \cdots \mathrm{ad}_{\hat{u}^{(k)}}^{j_{k}}, \\
S^{(k)} & =\sum_{\substack{j \in \pi(k+1) \\
j_{k+1}=0}} \frac{1}{j_{1} ! \cdots j_{k} !} \mathrm{ad}_{\hat{u}^{(k)}}^{j_{k}} \cdots \mathrm{ad}_{\hat{u}^{(1)}}^{j_{1}} .
\end{aligned}
$$

Note that since $\hat{v}$ is symmetric, $\hat{u}^{(1)}$ is antisymmetric so $S^{(1)} \hat{d}+Q^{(1)} \hat{v}$ is symmetric and it follows in turn that $\hat{u}^{(2)}$ is antisymmetric. Continuing in this way, we establish inductively that all $\hat{u}^{(k)}$ are antisymmetric and all $S^{(k)} \hat{d}$ and all $Q^{(k)} \hat{v}$ are symmetric operators. For $n_{1} \geq 1$, we define

$$
\widetilde{h}=\widetilde{h}_{n_{1}}=\hat{d}+\sum_{k=1}^{n_{1}} \mu^{k} \mathcal{R}\left(S^{(k-1)} \hat{d}+Q^{(k-1)} \hat{v}\right) .
$$

The following proposition will be shown in Subsection IV C.

Proposition 4.1. Let us consider the formal series $R=\sum_{k \geq 0} \mu^{k} R^{(k)}$ of operators on $\mathcal{S}$. We have

1. $\hat{h}=\mathcal{T}_{n_{1}}\left(R \tilde{h}_{n_{1}}\right)$.

2. For every $\hat{f}=\sum_{k=0}^{n_{1}} \mu^{k} \hat{f}^{(k)}$ with $\hat{f}^{(k)} \in \mathcal{S}$ for all $k$, it holds that

$$
\operatorname{ad}_{\hat{h}}\left(\mathcal{T}_{n_{1}}(R \hat{f})\right)=\mathcal{T}_{n_{1}}\left(R \operatorname{ad}_{\tilde{h}_{n_{1}}} \hat{f}\right)+\mu^{n_{1}+1} \operatorname{ad}_{\hat{v}} \sum_{k=0}^{n_{1}} R^{\left(n_{1}-k\right)} \hat{f}^{(k)} .
$$

The resonant Hamiltonian $\widetilde{h}$ and the formal operator $R$ have several characteristics that are good to remember.

1. Both $\widetilde{h}$ and $R$ are expressed as power series in $\mu$, as is seen from (49) and from the definition of $R$ given in Proposition 4.1. We introduce also the notation

$$
\widetilde{h}=\sum_{k=0}^{n_{1}} \mu^{k} \widetilde{h}^{(k)} \quad \text { with } \widetilde{h}^{(0)}=\hat{d} \text { and } \widetilde{h}^{(k)}=\mathcal{R}\left(S^{(k-1)} \hat{d}+Q^{(k-1)} \hat{v}\right) \text { for } k \geq 1 .
$$


2. For each $k \geq 0$, the operator $\widetilde{h}^{(k)}$ is an element of $\mathcal{S}$, and $R^{(k)}$ is an operator on $\mathcal{S}$. Let $\hat{f}=\sum_{x \in \mathbb{Z}_{N}} \hat{f}_{x} \in \mathcal{S}$ be given. The operators $\widetilde{h}^{k}$ and $R^{(k)} \hat{f}$ can be decomposed as a sum of local terms with, for example, for $k \geq 1$,

$$
\widetilde{h}_{x}^{(k)}=\mathcal{R}\left(S^{(k-1)} \hat{d}_{x}+Q^{(k-1)} \hat{v}_{x}\right) \text { and }\left(R^{(k)} \hat{f}\right)_{x}=R^{(k)} \hat{f}_{x} .
$$

Moreover, we will show in Subsection IV C that there exists an integer $r_{k}$ such that

$$
r\left(\widetilde{h}^{(k)}\right) \leq r_{k} \text { and } r\left(R^{(k)} \hat{f}\right) \leq r_{k}+r(f),
$$

where $r$ is the parameter introduced in the definition of the vector space $\mathcal{S}$.

3. The operators $h^{(k)}$ and $R^{(k)}$ depend on $\delta$ if $k \geq 1$. Let thus $k \geq 1$. In what follows, we will use the symbol $\hat{A}$ for polynomials of reduced annihilation and creation operators, the symbol $b$ to denote smooth, bounded functions on $\mathbb{R}^{N}$ with bounded derivatives of all orders, and the symbol $\hat{b}$ to denote the diagonal operator associated with a function of the form $\eta \mapsto b\left(\delta^{\gamma} \eta\right)$. We refer to diagonal operators $\hat{b}$ of this form as smooth diagonal operators. We will show the following assertions in Subsection IV C. First, there is an integer $m_{k}$ such that, given $x \in \mathbb{Z}_{N}, \widetilde{h}_{x}^{(k)}$ can be expressed as a sum of the type

$$
\widetilde{h}_{x}^{(k)}=\delta^{-2(k-1) \gamma^{\prime}} \sum_{j=1}^{m_{k}} \hat{A}_{j, x} \hat{b}_{j, x},
$$

where $0<\gamma^{\prime}=1-\gamma<1 / 2$ and such that $s\left(\hat{A}_{j, x}\right)$ and $s\left(\hat{b}_{j, x}\right)$ are subsets of $s\left(\widetilde{h}_{x}^{(k)}\right)$ and the bounds on the functions $b_{j, x}$ and its derivatives can be chosen uniformly in $x$.

Second, consider an operator $\hat{g} \in \mathcal{S}$ such that $\hat{g}_{x}=\hat{f}_{x} \hat{b}$ with $\hat{f}=\sum_{x \in \mathbb{Z}_{N}} \hat{f}_{x} \in \mathcal{S}$. Then there is an integer $m_{k, g}$ such that $\left(R^{(k)} \hat{g}\right)_{x}$ can be expressed as a sum of the type

$$
\left(R^{(k)} \hat{g}\right)_{x}=\delta^{-2 k \gamma^{\prime}} \sum_{j=1}^{m_{k, g}} \hat{A}_{j, x} \hat{b}_{j, x}
$$

such that $s\left(\hat{A}_{j, x}\right)$ and $s\left(\hat{b}_{j, x}\right)$ are subsets of $s\left(\widetilde{h}_{x}^{(k)}\right)$ and the bounds on the functions $b_{j, x}$ and its derivatives can be chosen uniformly in $x$.

\section{Proof of Proposition 4.1 and relations (53)-(55)}

Proof of Proposition 4.1. Given an operator $\hat{u} \in \mathcal{S}$, a formal transformation, seen as an operator on $\mathcal{S}$, is defined through

$$
\mathrm{e}^{\mu \mathrm{ad}_{\hat{u}}}=\sum_{k \geq 0} \frac{\mu^{k}}{k !} \operatorname{ad}_{\hat{u}^{k}} .
$$

Given a sequence $\left(\hat{u}^{(k)}\right)_{k \geq 1} \subset \mathcal{S}$, that we will later identify with the sequence defined by (45), we construct the formal unitary transformation

$$
\begin{aligned}
Q & =\cdots \mathrm{e}^{\mu^{n} \operatorname{ad}_{\hat{u}}(n)} \cdots \mathrm{e}^{\mu^{2} \operatorname{ad}_{\hat{u}^{(2)}}} \mathrm{e}^{\mu \mathrm{ad}_{\hat{u}}^{(1)}}=\sum_{j_{1} \geq 0, \ldots, j_{n} \geq 0, \cdots} \frac{\mu^{j_{1}+\cdots+n j_{n}+\cdots}}{j_{1} ! \cdots j_{n} ! \cdots}\left(\cdots \mathrm{ad}_{\hat{u}^{(n)}}^{j_{n}} \cdots \mathrm{ad}_{\hat{u}^{(1)}}^{j_{1}}\right) \\
& =\mathrm{Id}+\sum_{k \geq 1} \mu^{k} \sum_{j \in \pi(k)} \frac{1}{j_{1} ! \cdots j_{k} !} \mathrm{ad}_{\hat{u}^{(k)}}^{j_{k}} \cdots \mathrm{ad}_{\hat{u}^{(1)}}^{j_{1}}=\sum_{k \geq 0} \mu^{k} Q^{(k)} .
\end{aligned}
$$

The formal inverse of $Q$ is given by

$$
\begin{aligned}
R & =\mathrm{e}^{-\mu \operatorname{ad}_{\hat{u}^{(1)}}} \mathrm{e}^{-\mu^{2} \operatorname{ad}_{\hat{u}^{(2)}}} \cdots \mathrm{e}^{-\mu^{n} \mathrm{ad}_{\hat{u}^{(n)}}} \cdots \\
& =\mathrm{Id}+\sum_{k \geq 1} \mu^{k} \sum_{j \in \pi(k)} \frac{(-1)^{j_{1}+\cdots+j_{k}}}{j_{1} ! \cdots j_{k} !} \mathrm{ad}_{\hat{u}^{(1)}}^{j_{1}} \cdots \mathrm{ad}_{\hat{u}^{(k)}}^{j_{k}}=\sum_{k \geq 0} \mu^{k} R^{(k)} .
\end{aligned}
$$

Let us show the first part of Proposition 4.1. The operators $Q$ and $R$ are formal inverses of each other so that, for every $\hat{f} \in \mathcal{S}$ such that $\hat{f}=\mathcal{T}_{n_{1}} f$, it holds that

$$
\hat{f}=\mathcal{T}_{n_{1}}\left(R \mathcal{T}_{n_{1}}(Q \hat{f})\right),
$$


as can be checked by a direct computation with formal series. We will thus be done if we show that

$$
\widetilde{h}_{n_{1}}=\mathcal{T}_{n_{1}}(Q \hat{h}) \text {. }
$$

We compute

$$
Q \hat{h}=\sum_{k \geq 0} \mu^{k} Q^{(k)}(\hat{d}+\mu \hat{v})=\hat{d}+\sum_{k \geq 1} \mu^{k}\left(Q^{k} \hat{d}+Q^{(k-1)} \hat{v}\right)
$$

It holds that

$$
Q^{(k)}=S^{(k-1)}+\operatorname{ad}_{\hat{u}^{(k)}} \quad \text { for } \quad k \geq 1 .
$$

Since $\operatorname{ad}_{\hat{u}^{(k)}} \hat{d}=-\operatorname{ad}_{\hat{d}} \hat{u}^{(k)}$ for every $k \geq 1$, and taking now $\hat{u}^{(k)}$ as defined by (45), we obtain

$$
Q \hat{h}=\hat{d}+\sum_{k \geq 1} \mu^{k}\left(S^{(k-1)} \hat{d}-\operatorname{ad}_{\hat{d}} \hat{u}^{(k)}+Q^{(k-1)} \hat{v}\right)=\hat{d}+\sum_{k \geq 1} \mu^{k} \mathcal{R}\left(S^{(k-1)} \hat{d}+Q^{(k-1)} \hat{v}\right) .
$$

From this, we derive (60).

Let us then show the second part of Proposition 4.1. The operators $Q$ and $R$ are formal unitary transformations, inverse of each other. Therefore

$$
\operatorname{ad}_{\hat{h}} R=R \operatorname{ad}_{Q \hat{h}}
$$

as a direct but lengthy computation with formal series can confirm. Let us next take $\hat{f} \in \mathcal{S}$ such that $\hat{f}=\mathcal{T}_{n_{1}}(\hat{f})$. By $(60)$, we find that

$$
\mathcal{T}_{n_{1}}\left(R \operatorname{ad}_{\tilde{h}} \hat{f}\right)=\mathcal{T}_{n_{1}}\left(R \operatorname{ad}_{\mathcal{T}_{n_{1}}(Q \hat{h})} \hat{f}\right)=\mathcal{T}_{n_{1}}\left(R \operatorname{ad}_{Q \hat{h}} \hat{f}\right)
$$

since higher order terms do not contribute due to the overall truncation $\mathcal{T}_{n_{1}}$. Therefore, by (64),

$$
\begin{aligned}
\operatorname{ad}_{\hat{h}}\left(\mathcal{T}_{n_{1}}(R \hat{f})\right)-\mathcal{T}_{n_{1}}\left(R \operatorname{ad}_{\tilde{h}} \hat{f}\right) & =\operatorname{ad}_{\hat{h}}\left(\mathcal{T}_{n_{1}}(R \hat{f})\right)-\mathcal{T}_{n_{1}}\left(R \operatorname{ad}_{Q \hat{h}} \hat{f}\right) \\
& =\operatorname{ad}_{\hat{h}}\left(\mathcal{T}_{n_{1}}(R \hat{f})\right)-\mathcal{T}_{n_{1}}\left(\operatorname{ad}_{\hat{h}} R \hat{f}\right) .
\end{aligned}
$$

Since $\operatorname{ad}_{\hat{h}}=\operatorname{ad}_{\hat{d}}+\mu \operatorname{ad}_{\hat{v}}$, it is finally computed that

$$
\operatorname{ad}_{\hat{h}}\left(\mathcal{T}_{n_{1}}(R \hat{f})\right)-\mathcal{T}_{n_{1}}\left(\operatorname{ad}_{\hat{h}} R \hat{f}\right)=\mu^{n_{1}+1} \operatorname{ad}_{\hat{v}} \sum_{k=0}^{n_{1}} R^{\left(n_{1}-k\right)} \hat{f}^{(k)} .
$$

This completes the proof.

Proof of (53)-(55). Given two operators $\hat{f}, \hat{g} \in \mathcal{S}$, we can decompose $\operatorname{ad}_{\hat{f}} \hat{g}$ as a sum of local terms $\left(\operatorname{ad}_{\hat{f}} g\right)_{x}=\operatorname{ad}_{\hat{f}} \hat{g}_{x}$ so that $r\left(\operatorname{ad}_{\hat{f}} \hat{g}\right) \leq 2 r(\hat{f})+r(\hat{g})$. If we write $\hat{u}=\operatorname{ad}_{\hat{d}}^{-1}(\mathrm{Id}-\mathcal{R}) \hat{f}$ for the solution to

$$
\operatorname{ad}_{\hat{d}} \hat{u}=(\mathrm{Id}-\mathcal{R}) \hat{f}
$$

given by (42), then we see that $r\left(\operatorname{ad}_{\hat{d}}^{-1}(\operatorname{Id}-\mathcal{R}) \hat{f}\right)=r(\hat{f})$. From this equation and (46)-(48), we readily deduce (53).

Let us now show (54) and (55). Since we are only interested in tracking the dependence on $\delta$ we may simplify notation as much as possible in the following way. We use symbols $\hat{A}$ and $\hat{b}$ with the same meanings as in the paragraph where (54) and (55) are stated. Let $n \geq 0$. For $\hat{g} \in \mathcal{S}$, we write $\hat{g} \sim \delta^{-n}$ if $\hat{g}=\sum_{x \in \mathbb{Z}_{N}} \hat{g}_{x}$ with

$$
\hat{g}_{x}=\delta^{-n} \sum_{j} \hat{A}_{\rho_{j}} \hat{b}_{j, x}
$$

with all bounds on the functions $b_{j, x}$ and their derivatives uniform in $x$.

For operators $B$ on $\mathcal{S}$, we write $B \sim \delta^{-n}$ if for any $\hat{f} \in \mathcal{S}$ such that $\hat{f} \sim \delta^{-m}$ we have $B \hat{f} \sim \delta^{-n-m}$.

We now observe that if $\hat{g} \sim \delta^{-n}$ and $\hat{f} \sim \delta^{-m}$ then $\operatorname{ad}_{\hat{g}} \hat{f} \sim \delta^{-(n+m)+\gamma}$. Indeed, $\operatorname{ad}_{\hat{g}} \hat{f}$ is a sum whose terms take the following form:

$$
\begin{aligned}
\delta^{-(n+m)}\left[\hat{A} \rho \hat{b}, \hat{A}_{\rho^{\prime}} \hat{b}^{\prime}\right] & =\delta^{-(n+m)}\left(\hat{A}_{\rho} \hat{A}_{\rho^{\prime}}\left(\Delta_{\rho^{\prime}} \hat{b}\right) \hat{b}^{\prime}+\left[\hat{A}_{\rho}, \hat{A}_{\rho^{\prime}}\right] \hat{b}^{\prime} \hat{b}+\hat{A}_{\rho^{\prime}} \hat{A}_{\rho}\left(\Delta_{\rho} \hat{b}^{\prime}\right) \hat{b}\right) \\
& =\delta^{-(n+m)+\gamma} \sum_{i=1}^{l} \hat{A}_{i, \rho+\rho^{\prime}} \hat{b}_{i}
\end{aligned}
$$


for some number $l$. The last step is obtained by noticing that discrete derivatives of the smooth diagonal operators have matrix elements of order $\delta^{\gamma}$ and commutators of monomials of reduced annihilation operators can always be written as $\delta$ times a polynomial in the reduced annihilation and creation operators whose terms all affect the move obtained by summing the moves of the commutants.

The diagonal operators $\hat{b}_{i}$ are products of smooth diagonal operators and discrete derivatives of smooth diagonal operators and are therefore themselves smooth.

We observe also that if $\hat{u}$ is the solution to the equation

$$
\operatorname{ad}_{\hat{d}} \hat{u}=(\mathrm{Id}-\mathcal{R}) \hat{f}
$$

with $\hat{f} \sim \delta^{-n}$, then since we can take

$$
\hat{u}_{x}=\delta^{-2} \sum_{\rho \in M_{r(f)}} \hat{f}_{x}^{(\rho)} \frac{\mathbb{1}-\hat{\zeta}_{\rho}}{\Delta_{\rho} \hat{E}}=\delta^{-n-2+\gamma} \sum_{\rho \in M_{r(f)}} \sum_{j} \hat{A}_{\rho_{j}} \hat{b}_{j} \frac{\mathbb{1}-\hat{\zeta}_{\rho}}{\delta^{\gamma} \Delta_{\rho} \hat{E}},
$$

where the operators

$$
\hat{b}_{j} \frac{\mathbb{1}-\hat{\zeta}_{\rho}}{\delta^{\gamma} \Delta_{\rho} \hat{E}}
$$

are smooth diagonal operators, we have $\hat{u} \sim \delta^{-n-2+\gamma}$.

Remembering that we defined $\gamma^{\prime}=1-\gamma$, let us now establish recursively that for $k \geq 1$, we have

$$
Q^{(k-1)}, R^{(k-1)}, S^{(k-1)} \hat{d} \sim \delta^{-2(k-1) \gamma^{\prime}}, \quad \text { and } \quad \hat{u}^{(k)} \sim \delta^{-2 k \gamma^{\prime}-\gamma} .
$$

It is easily checked from the definitions that these relations hold for $k=1$. Let us see that the claim for $1, \ldots, k \geq 1$ implies the claim for $k+1$.

First, from the definitions (46) and (47) and the fact that if $j \in \pi(k)$ then $j_{1}+2 j_{2}+\cdots+k j_{k}=k$, we obtain

$$
Q^{(k)}, R^{(k)} \sim\left(\delta^{-2 k \gamma^{\prime}-\gamma+\gamma}\right)^{j_{k}} \cdots\left(\delta^{-2 \gamma^{\prime}-\gamma+\gamma}\right)^{j_{1}}=\delta^{-2 \gamma^{\prime} \sum_{l} l j_{l}}=\delta^{-2 k \gamma^{\prime}} .
$$

Let us then treat $S^{(k)} \hat{d}$. We decompose $S^{(k)} \hat{d}=\sum_{j \in \pi(k+1)} S_{j}^{(k)} \hat{d}$. Fixing $\underline{j}_{-} \in \pi(k+1)$ and letting $l \geq 1$ be the smallest integer such that $j_{l} \geq 1$; we get for some constant $C(j)$ that

$$
\begin{aligned}
S_{\underline{j}}^{(k)} & =C(j) \operatorname{ad}_{\hat{u}^{(k)}}^{j_{k}} \cdots \operatorname{ad}_{\hat{u}^{(l)}}^{j_{l}-1}\left(\operatorname{ad}_{\hat{u}^{(l)}} d\right) \\
& =-C(j) \operatorname{ad}_{\hat{u}_{k}^{(k)}}^{j_{k}} \cdots \operatorname{ad}_{\hat{u}^{\prime l)}}^{j_{l}-1}\left((\operatorname{Id}-\mathcal{R})\left(S^{(l-1)} \hat{d}+Q^{l-1} \hat{v}\right)\right) \\
& \sim\left(\delta^{-2 k \gamma^{\prime}}\right)^{j_{k}} \cdots\left(\delta^{-2 l \gamma^{\prime}}\right)^{j_{l}} \delta^{2 l \gamma^{\prime}} \delta^{-2(l-1) \gamma^{\prime}} \\
& =\delta^{-2 k \gamma^{\prime}} .
\end{aligned}
$$

Finally, we have from the definition (45) and the induction hypothesis that

$$
\hat{u}^{(k+1)} \sim \delta^{-2 k \gamma^{\prime}-2+\gamma}=\delta^{-2(k+1) \gamma^{\prime}-\gamma}
$$

as required.

It is instructive to couple back to the Introduction and see that the Bose-Hubbard model is indeed a critical case in the sense described there, i.e., we want to see explicitly that the generator of the transformation $Q$ constructed above is not small in $\mu$. From (58), we see that the leading contribution to the generator is $\mu \hat{u}^{(1)}$. Let us write the reduced hopping $\hat{v}=\sum_{\rho} \hat{v}^{(\rho)}$ as a sum over nearest neighbour hoppings, and it then follows from (42) and (45) that

$$
\hat{u}^{(1)}=\mu^{-2} \sum_{\rho} \hat{v}^{(\rho)} \frac{\left(\mathbb{1}-\hat{\zeta}_{\rho}\right)}{\Delta_{\rho} \hat{E}},
$$

where we also put $\delta=\mu$ because we are dealing with the Bose-Hubbard model. For a typical state with respect to the Gibbs state, we have $v^{(\rho)} \sim g$ and $\Delta_{\rho} \hat{E} \sim \mu^{-1}$. Therefore $\mu \hat{u}^{(1)} \sim g$, i.e., the leading contribution to the generator is a constant and is not small in the limit $\mu \rightarrow 0$. 


\section{GEOMETRY OF RESONANCES}

Given a point $x \in \mathbb{Z}_{N}$ and considering the phase space as a subset of $\mathbb{R}^{N}$, we construct a subset $\mathrm{R}(x)$ of $\mathbb{R}^{N}$ with the two following characteristics. First, if a point of the phase space does not belong to this set, then the energy current for the Hamiltonian $\widetilde{h}$ vanishes through the bonds near $x$. Second, it is approximately invariant under the dynamics generated by $\widetilde{h}$, meaning that the commutator of $\widetilde{h}$ and the projector on the space spanned by states in the set vanish everywhere except on a subspace spanned by classical states in a subset $\mathrm{S}(x) \subset \mathbb{R}^{N}$ which is of small probability with respect to the Gibbs state.

The ideas of this section are best understood visually. We hope that Fig. 1 will help in that respect. We let

$$
r=r\left(n_{1}\right)=\max _{1 \leq k \leq n_{1}} r_{k},
$$

where the numbers $r_{k}$ are defined in (53). We let $\delta>0$ be as in Sec. IV.

\section{A. Preliminary definitions}

We recall that, given $\rho \in \mathbb{Z}^{N}$, we denote by $\operatorname{Supp}(\rho) \subset \mathbb{Z}^{N}$ the set of points $x$ such that $\rho_{x} \neq 0$ and we have defined the set $M_{r} \subset \mathbb{Z}^{N}$ of vectors $\rho=\left(\rho_{x}\right)_{x \in \mathbb{Z}_{N}}$ such that $\max _{x \in \mathbb{Z}_{N}}\left|\rho_{x}\right| \leq r$ and $\operatorname{Supp}(\rho)$ $\subset \mathrm{B}(x, r)$ for some $\mathrm{x}$ in $\mathbb{Z}_{N}$. We write $|\rho|_{2}^{2}=\sum_{x}\left|\rho_{x}\right|^{2}$. One easily checks that for any $\rho \in M_{r}$ and $r>1$, we have $|\rho|_{2} \leq 2 r^{2}$ and this will be used without further comment.

Given $x \in \mathbb{Z}^{d}$, we say that a subset $\left\{\rho_{1}, \ldots, \rho_{p}\right\} \subset M_{r}$ is a cluster around $x$ if

1. the vectors $\rho_{1}, \ldots, \rho_{p}$ are linearly independent;

2. if $p \geq 2$, for all $1 \leq i \neq j \leq p$, there exist $1 \leq i_{1}, \ldots, i_{m} \leq p$ such that $i_{1}=i, i_{m}=j$, and $\operatorname{Supp}\left(\rho_{i_{s}}\right) \cap \operatorname{Supp}\left(\rho_{i_{s+1}}\right) \neq \varnothing$ for all $1 \leq s \leq m-1$;

3. $\operatorname{Supp}\left(\rho_{j}\right) \subset \mathrm{B}(x, 4 r)$ for some $1 \leq j \leq p$.

Finally, given $\rho \in M_{r}$, we define

$$
\pi(\rho)=\left\{\eta \in \mathbb{R}^{N}: \rho \cdot \eta=0\right\} .
$$

Given a subspace $E \subset \mathbb{R}^{N}$, and given $\eta \in \mathbb{R}^{N}$, we denote by $P(\eta, E)$ the orthogonal projection of $\eta$ on the subspace $E$.

\section{B. Approximately invariant resonant zones}

Let $L>0$, let $n_{2} \geq 1$, and let $x \in \mathbb{Z}_{N}$. Let us define two subsets of $\mathbb{R}^{N}$ : a set $\mathrm{R}_{\delta, n_{2}}(x) \subset \mathbb{R}^{N}$ of resonant points, and a small set $\mathrm{S}_{\delta, n_{2}}(x) \subset \mathbb{R}^{N}$ of "multi-resonant" points.

To define $\mathrm{R}_{\delta, n_{2}}(x)$, let us first define the sets $\mathrm{B}_{\delta}\left(\rho_{1}, \ldots, \rho_{p}\right) \subset \mathbb{R}^{N}$, where $\left\{\rho_{1}, \ldots, \rho_{p}\right\}$ is a cluster around $x$. We say that $\eta \in \mathrm{B}_{\delta}\left(\rho_{1}, \ldots, \rho_{p}\right)$ if

$$
\left|\eta-P\left(\eta, \pi\left(\rho_{1}\right) \cap \cdots \cap \pi\left(\rho_{p}\right)\right)\right|_{2} \leq L^{p} \delta^{-\gamma}
$$

with $\gamma$ as in Sec. IV and if, for every linearly independent $\rho_{1}^{\prime}, \ldots, \rho_{p^{\prime}}^{\prime} \in M_{r} \cap \operatorname{span}\left\{\rho_{1}, \ldots, \rho_{p}\right\}$,

$$
\left|P\left(\eta, \pi\left(\rho_{1}^{\prime}\right) \cap \cdots \cap \pi\left(\rho_{p^{\prime}}^{\prime}\right)\right)-P\left(\eta, \pi\left(\rho_{1}\right) \cap \cdots \cap \pi\left(\rho_{p}\right)\right)\right|_{2} \leq\left(L^{p}-L^{p^{\prime}}\right) \delta^{-\gamma} .
$$

We next define $\mathrm{R}_{\delta, n_{2}}(x)$ as the union of all the sets $\mathrm{B}_{\delta}\left(\rho_{1}, \ldots, \rho_{p}\right) \subset \mathbb{R}^{N}$ with $p \leq n_{2}$.

We then define $\mathrm{S}_{\delta, n_{2}}(x)$ as the set of points $\eta \in \mathbb{R}^{N}$ for which there exists a cluster $\left\{\rho_{1}, \ldots, \rho_{n_{2}}\right\}$ around $x$, such that $\left|\rho_{j} \cdot \eta\right| \leq L^{n_{2}+1} \delta^{-\gamma}$ for every $1 \leq j \leq n_{2}$.

We finally define a smooth indicator function on the complement of $\mathrm{R}_{\delta, n_{2}}(x)$ by means of a convolution,

$$
\theta_{x, \delta, n_{2}}(\eta)=1-\frac{1}{\left(\int_{\mathbb{R}} \xi_{\delta^{-\gamma}}(z) \mathrm{d} z\right)^{N}} \int_{\mathbb{R}^{N}} \chi_{\mathrm{R}_{\delta, n_{2}(x)}}\left(\eta+\eta^{\prime}\right)\left(\prod_{x \in \mathbb{Z}_{N}} \xi_{\delta^{-\gamma}}\left(\eta_{x}^{\prime}\right)\right) \mathrm{d} \eta^{\prime} .
$$

Proposition 5.1. Let $n_{1}$ be given, and so $r\left(n_{1}\right)$ defined by (76) be fixed as well. Let then $n_{2} \geq 1$ be fixed. The following holds for L large enough and $\delta$ and $\mu$ small enough: 
1. If $\theta_{x, \delta, n_{2}}(\eta)>0$, then $\zeta_{\rho}(\eta)=0$ for all $\rho \in M_{r}$ such that $\operatorname{Supp}(\rho) \subset \mathrm{B}(x, 4 r)$.

2. $\left(\operatorname{ad}_{\tilde{h}} \hat{\theta}_{x, \delta, n_{2}}\right) \hat{P}_{\eta}=0$ for all $\eta \in \Omega_{N} \subset \mathbb{R}^{N}$ such that $\eta \notin \mathrm{S}_{n_{2}}(x)$.

\section{Proof of Proposition 5.1}

We start by a series of lemmas. The first one simply expresses, in a particular case, that if a point is close to two vector spaces, then it is also close to their intersection. The uniformity of the constant $\mathrm{C}$ comes from the fact that we impose the vectors to sit in the set $M_{r}$.

Lemma 5.2. Let $p \geq 1$. There exists a constant $\mathrm{C}=\mathrm{C}(r, p)<+\infty$ such that, given linearly independent vectors $\rho_{1}, \ldots, \rho_{p}, \rho_{p+1} \in M_{r}$, and given $\eta \in \mathbb{R}^{N}$, it holds that

$$
\left|\eta-P\left(\eta, \pi\left(\rho_{1}\right) \cap \cdots \cap \pi\left(\rho_{p}\right) \cap \pi\left(\rho_{p+1}\right)\right)\right|_{2} \leq \mathrm{C}\left(\left|\rho_{p+1} \cdot \eta\right|+\left|\eta-P\left(\eta, \pi\left(\rho_{1}\right) \cap \cdots \cap \pi\left(\rho_{p}\right)\right)\right|_{2}\right) .
$$

Proof. First,

$$
\begin{aligned}
\left|\eta-P\left(\eta, \pi\left(\rho_{1}\right) \cap \cdots \cap \pi\left(\rho_{p+1}\right)\right)\right|_{2} & \leq\left|\eta-P\left(\eta, \pi\left(\rho_{1}\right) \cap \cdots \cap \pi\left(\rho_{p}\right)\right)\right|_{2} \\
& +\left|P\left(\eta, \pi\left(\rho_{1}\right) \cap \cdots \cap \pi\left(\rho_{p+1}\right)\right)-P\left(\eta, \pi\left(\rho_{1}\right) \cap \cdots \cap \pi\left(\rho_{p}\right)\right)\right|_{2} .
\end{aligned}
$$

The lemma is already shown if the second term in the right-hand side is zero. We further assume this not to be the case. Next, since $\rho_{p+1} \cdot P\left(\eta, \pi\left(\rho_{1}\right) \cap \cdots \cap \pi\left(\rho_{p+1}\right)\right)=0$, we obtain

$$
\begin{aligned}
& \rho_{p+1} \cdot \eta=\rho_{p+1} \cdot\left(\eta-P\left(\eta, \pi\left(\rho_{1}\right)\right.\right.\left.\left.\cap \cdots \cap \pi\left(\rho_{p}\right)\right)\right)+ \\
& \rho_{p+1} \cdot\left(P\left(\eta, \pi\left(\rho_{1}\right) \cap \cdots \cap \pi\left(\rho_{p}\right)\right)-P\left(\eta, \pi\left(\rho_{1}\right) \cap \cdots \cap \pi\left(\rho_{p+1}\right)\right)\right) .
\end{aligned}
$$

This implies

$$
\begin{aligned}
\left|\rho_{p+1} \cdot\left(P\left(\eta, \pi\left(\rho_{1}\right) \cap \cdots \cap \pi\left(\rho_{p}\right)\right)-P\left(\eta, \pi\left(\rho_{1}\right) \cap \cdots \cap \pi\left(\rho_{p+1}\right)\right)\right)\right| \leq \\
\left|\rho_{p+1} \cdot \eta\right|+\left|\rho_{p+1}\right|_{2}\left|\eta-P\left(\eta, \pi\left(\rho_{1}\right) \cap \cdots \cap \pi\left(\rho_{p}\right)\right)\right|_{2} .
\end{aligned}
$$

The vector

$$
v=\frac{P\left(\eta, \pi\left(\rho_{1}\right) \cap \cdots \cap \pi\left(\rho_{p}\right)\right)-P\left(\eta, \pi\left(\rho_{1}\right) \cap \cdots \cap \pi\left(\rho_{p+1}\right)\right)}{\left|P\left(\eta, \pi\left(\rho_{1}\right) \cap \cdots \cap \pi\left(\rho_{p}\right)\right)-P\left(\eta, \pi\left(\rho_{1}\right) \cap \cdots \cap \pi\left(\rho_{p+1}\right)\right)\right|_{2}}
$$

is well defined since we have assumed that the denominator in this expression does not vanish. The bound (80) is rewritten as

$$
\begin{aligned}
&\left|P\left(\eta, \pi\left(\rho_{1}\right) \cap \cdots \cap \pi\left(\rho_{p}\right)\right)-P\left(\eta, \pi\left(\rho_{1}\right) \cap \cdots \cap \pi\left(\rho_{p+1}\right)\right)\right|_{2} \leq \\
& \frac{\left|\rho_{p+1} \cdot \eta\right|+\left|\rho_{p+1}\right| 2\left|\eta-P\left(\eta, \pi\left(\rho_{1}\right) \cap \cdots \cap \pi\left(\rho_{p}\right)\right)\right|_{2}}{\left|\rho_{p+1} \cdot v\right|} .
\end{aligned}
$$

Inserting this last inequality in (79), we arrive at

$$
\left|\eta-P\left(\eta, \pi\left(\rho_{1}\right) \cap \cdots \cap \pi\left(\rho_{p+1}\right)\right)\right|_{2} \leq \frac{\left|\rho_{p+1} \cdot \eta\right|}{\left|\rho_{p+1} \cdot v\right|}+\left(1+\frac{\left|\rho_{p+1}\right|_{2}}{\left|\rho_{p+1} \cdot v\right|}\right)\left|\eta-P\left(\eta, \pi\left(\rho_{1}\right) \cap \cdots \cap \pi\left(\rho_{p}\right)\right)\right|_{2} .
$$

To finish the proof, it remains to establish that $\left|\rho_{p+1} \cdot v\right|$ can be bounded from below by some strictly positive constant, where $v$ is given by (81). Let us show that

$$
v= \pm \frac{P\left(\rho_{p+1}, \pi\left(\rho_{1}\right) \cap \cdots \cap \pi\left(\rho_{p}\right)\right)}{\left|P\left(\rho_{p+1}, \pi\left(\rho_{1}\right) \cap \cdots \cap \pi\left(\rho_{p}\right)\right)\right|_{2}} .
$$

We can find vectors $\rho_{p+2}, \ldots, \rho_{N}$ so that $\left\{\rho_{1}, \ldots, \rho_{N}\right\}$ forms a basis of $\mathbb{R}^{N}$ and so that every vector $\rho_{j}$ with $p+2 \leq j \leq N$ is orthogonal to $\operatorname{span}\left\{\rho_{1}, \ldots, \rho_{p+1}\right\}$. We express the vector $\eta$ in this basis, $\eta=\sum_{j=1}^{N} \eta^{j} \rho_{j}$, and, from (81), we deduce that, for some non-zero constant $R$, we have

$$
v=R \sum_{j=1}^{N} \eta^{j}\left\{P\left(\rho_{j}, \pi\left(\rho_{1}\right) \cap \cdots \cap \pi\left(\rho_{p}\right)\right)-P\left(\rho_{j}, \pi\left(\rho_{1}\right) \cap \cdots \cap \pi\left(\rho_{p+1}\right)\right)\right\} .
$$


All the terms corresponding to $1 \leq j \leq p$ vanish since $\rho_{j} \perp \pi\left(\rho_{j}\right)$, and the term $P\left(\rho_{p+1}, \pi\left(\rho_{1}\right) \cap \cdots \cap\right.$ $\left.\pi\left(\rho_{p+1}\right)\right)$ vanishes for the same reason, and all the terms corresponding to $j \geq p+2$ vanish too, as they read in fact $\rho_{j}-\rho_{j}=0$. So, the only term left is $R \eta^{p+1} P\left(\rho_{p+1}, \pi\left(\rho_{1}\right) \cap \cdots \cap \pi\left(\rho_{p}\right)\right)$ and, since $|v|=1$, we arrive at (82).

From (82), we deduce that

$$
\left|v \cdot \rho_{p+1}\right|=\left|P\left(\rho_{p+1}, \pi\left(\rho_{1}\right) \cap \cdots \cap \pi\left(\rho_{p}\right)\right)\right|_{2} .
$$

If $\rho_{p+1} \perp \operatorname{span}\left\{\rho_{1}, \ldots, \rho_{p}\right\}$, then the right-hand side just becomes $\left|\rho_{p+1}\right|_{2}$. This quantity is bounded from below by a strictly positive constant since so is the norm of any non-zero vector in $M_{r}$. Otherwise, if $\rho_{p+1} \mathcal{L} \operatorname{span}\left\{\rho_{1}, \ldots, \rho_{p}\right\}$, we know however that the quantity cannot vanish since $\rho_{p+1} \notin \operatorname{span}\left\{\rho_{1}\right.$, $\left.\ldots, \rho_{p}\right\}$. Because they are only finitely many vectors $\rho \in M r$ with the property that $\rho \not \operatorname{span}\left\{\rho_{1}, \ldots\right.$, $\left.\rho_{p}\right\}$, we conclude that the quantity is bounded from below by a strictly positive constant.

The next lemma describes the crucial geometrical properties of the sets $\mathrm{B}_{\delta}\left(\rho_{1}, \ldots, \rho_{p}\right)$ which allows us to establish the second assertion of Proposition 5.1.

Lemma 5.3. Let $\left\{\rho_{1}, \ldots, \rho_{p}\right\}$ be a cluster around $x$. If, given $\mathrm{K}<+\infty, L$ is taken large enough, then, for every $\rho \in M_{r} \cap \operatorname{span}\left\{\rho_{1}, \ldots, \rho_{p}\right\}$, it holds that if

$$
\eta \in \mathrm{B}_{\delta}\left(\rho_{1}, \ldots, \rho_{p}\right) \text { and }|\rho \cdot \eta| \leq \mathrm{K} \delta^{-\gamma},
$$

then

$$
\eta+t \rho \in \mathrm{B}_{\delta}\left(\rho_{1}, \ldots, \rho_{p}\right) \quad \text { as longas }|t| \leq \delta^{-\gamma} .
$$

Proof. To simplify some further expressions, let us define

$$
\eta^{\prime}=\eta-P\left(\eta, \pi\left(\rho_{1}\right) \cap \cdots \cap \pi\left(\rho_{p}\right)\right) \in \operatorname{span}\left(\rho_{1}, \ldots, \rho_{p}\right) .
$$

The conditions ensuring that $\eta \in \mathrm{B}\left(\rho_{1}, \ldots, \rho_{p}\right)$ now simply read

$$
\left|\eta^{\prime}\right|_{2} \leq L^{p} \delta^{-\gamma} \quad \text { and } \quad\left|P\left(\eta^{\prime}, \pi\left(\rho_{1}^{\prime}\right) \cap \cdots \cap \pi\left(\rho_{p^{\prime}}^{\prime}\right)\right)\right|_{2} \leq\left(L^{p}-L^{p^{\prime}}\right) \delta^{-\gamma} \quad\left(p^{\prime}<p\right),
$$

for all linearly independent $\rho_{1}^{\prime}, \ldots, \rho_{p \prime}^{\prime} \in M_{r} \cap \operatorname{span}\left\{\rho_{1}, \ldots, \rho_{p}\right\}$. The condition $|\rho \cdot \eta| \leq \mathrm{K} \delta^{-\gamma}$ implies $\left|\rho \cdot \eta^{\prime}\right| \leq \mathrm{K} \delta^{-\gamma}$. We need to show that

$$
\begin{array}{lrr}
\left|\eta^{\prime}+t \rho\right|_{2} \leq L^{p} \delta^{-\gamma} & \text { for } & |t| \leq \delta^{-\gamma}, \\
\left|P\left(\eta^{\prime}+t \rho, \pi\left(\rho_{1}^{\prime}\right) \cap \cdots \cap \pi\left(\rho_{p^{\prime}}^{\prime}\right)\right)\right|_{2} \leq\left(L^{p}-L^{p^{\prime}}\right) \delta^{-\gamma} & \text { for } & |t| \leq \delta^{-\gamma} .
\end{array}
$$

Let us start with (84),

$$
\begin{aligned}
\left|\eta^{\prime}+t \rho\right|_{2} & \leq\left|\eta^{\prime}-P\left(\eta^{\prime}, \pi(\rho)\right)\right|_{2}+\left|P\left(\eta^{\prime}, \pi(\rho)\right)\right|_{2}+|t||\rho|_{2} \leq\left|\eta^{\prime} \cdot \rho\right|+\left|P\left(\eta^{\prime}, \pi(\rho)\right)\right|_{2}+|t||\rho|_{2} \\
& \leq \mathrm{K} \delta^{-\gamma}+\left(L^{p}-L\right) \delta^{-\gamma}+2 r^{2} \delta^{-\gamma} \leq L^{p} \delta^{-\gamma} .
\end{aligned}
$$

Here, to get the penultimate inequality, we have used (83) and the hypothesis $\rho \in M_{r} \cap$ $\operatorname{span}\left\{\rho_{1}, \ldots, \rho_{p}\right\}$, implying in particular $|\rho|_{2} \leq 2 r^{2}$, while the last inequality is valid for large enough $L$.

Let us next move to (85). Let us fix $\rho_{1}^{\prime}, \ldots, \rho_{p^{\prime}}^{\prime}$. It is seen that, if $\rho \in \operatorname{span}\left\{\rho_{1}^{\prime}, \ldots, \rho_{p^{\prime}}^{\prime}\right\}$, then (85) is actually satisfied for all $t \in \mathbb{R}$. Let us therefore assume $\rho \notin \operatorname{span}\left\{\rho_{1}^{\prime}, \ldots, \rho_{p^{\prime}}^{\prime}\right\}$. We write also $\rho=\rho_{p^{\prime}+1}^{\prime}$. We will show that, because $|\rho \cdot \eta| \leq \mathrm{K} \delta^{-\gamma}$, then in fact

$$
\left|P\left(\eta^{\prime}, \pi\left(\rho_{1}^{\prime}\right) \cap \cdots \cap \pi\left(\rho_{p^{\prime}}^{\prime}\right)\right)\right|_{2} \leq\left(L^{p}-L^{p^{\prime}}-2 r^{2}\right) \delta^{-\gamma} .
$$

Since $|\rho|_{2} \leq 2 r^{2}$, this will imply (85).

To establish (86), we start by writing the decompositions

$$
\begin{aligned}
& \left|\eta^{\prime}\right|_{2}^{2}=\left|\eta^{\prime}-P\left(\eta^{\prime}, \pi\left(\rho_{1}^{\prime}\right) \cap \cdots \cap \pi\left(\rho_{p^{\prime}}^{\prime}\right)\right)\right|_{2}^{2}+\left|P\left(\eta^{\prime}, \pi\left(\rho_{1}^{\prime}\right) \cap \cdots \cap \pi\left(\rho_{p^{\prime}}^{\prime}\right)\right)\right|_{2}^{2}, \\
& \left|\eta^{\prime}\right|_{2}^{2}=\left|\eta^{\prime}-P\left(\eta^{\prime}, \pi\left(\rho_{1}^{\prime}\right) \cap \cdots \cap \pi\left(\rho_{p^{\prime}+1}^{\prime}\right)\right)\right|_{2}^{2}+\left|P\left(\eta^{\prime}, \pi\left(\rho_{1}^{\prime}\right) \cap \cdots \cap \pi\left(\rho_{p^{\prime}+1}^{\prime}\right)\right)\right|_{2}^{2} .
\end{aligned}
$$


We bound the first term in the right-hand side of (88) by applying Lemma 5.2 and then using (87),

$$
\begin{aligned}
\left|\eta^{\prime}-P\left(\eta^{\prime}, \pi\left(\rho_{1}^{\prime}\right) \cap \cdots \cap \pi\left(\rho_{p^{\prime}+1}^{\prime}\right)\right)\right|_{2}^{2} & \leq \mathrm{C}\left(\left|\rho \cdot \eta^{\prime}\right|^{2}+\left|\eta^{\prime}-P\left(\eta^{\prime}, \pi\left(\rho_{1}^{\prime}\right) \cap \cdots \cap \pi\left(\rho_{p^{\prime}}^{\prime}\right)\right)\right|_{2}^{2}\right) \\
& \leq \mathrm{C}\left(\left|\rho \cdot \eta^{\prime}\right|^{2}+\left|\eta^{\prime}\right|_{2}^{2}-\left|P\left(\eta^{\prime}, \pi\left(\rho_{1}^{\prime}\right) \cap \cdots \cap \pi\left(\rho_{p^{\prime}}^{\prime}\right)\right)\right|_{2}^{2}\right) .
\end{aligned}
$$

It may be assumed that $C \geq 1$. Reinserting this bound in (88) yields

$$
\begin{aligned}
\left|\eta^{\prime}\right|_{2}^{2} & \leq \mathrm{C}\left(\left|\rho \cdot \eta^{\prime}\right|^{2}+\left|\eta^{\prime}\right|_{2}^{2}-\left|P\left(\eta^{\prime}, \pi\left(\rho_{1}^{\prime}\right) \cap \cdots \cap \pi\left(\rho_{p^{\prime}}^{\prime}\right)\right)\right|_{2}^{2}\right)+\left|P\left(\eta^{\prime}, \pi\left(\rho_{1}^{\prime}\right) \cap \cdots \cap \pi\left(\rho_{p^{\prime}+1}^{\prime}\right)\right)\right|_{2}^{2} \\
& \leq \mathrm{C}\left(\mathrm{K}^{2} \delta^{-2 \gamma}+\left|\eta^{\prime}\right|_{2}^{2}-\left|P\left(\eta^{\prime}, \pi\left(\rho_{1}^{\prime}\right) \cap \cdots \cap \pi\left(\rho_{p^{\prime}}^{\prime}\right)\right)\right|_{2}^{2}\right)+\left(L^{p}-L^{p^{\prime}+1}\right)^{2} \delta^{-2 \gamma} .
\end{aligned}
$$

where the hypotheses $\left|\rho \cdot \eta^{\prime}\right| \leq \mathrm{K} \delta^{-\gamma}$ and $\eta \in \mathrm{B}\left(\rho_{1}, \ldots, \rho_{p}\right)$ have been used to get the last line.

Let us now show that (89) implies (86) for $L$ large enough. For this, let us write

$$
\begin{array}{rlrl}
\left|\eta^{\prime}\right|_{2} & =(1-\mu)^{1 / 2} L^{p} \delta^{-\gamma} \quad \text { with } & 0 \leq \mu \leq 1, & \\
\left|P\left(\eta^{\prime}, \pi\left(\rho_{1}^{\prime}\right) \cap \cdots \cap \pi\left(\rho_{p^{\prime}}^{\prime}\right)\right)\right|_{2} & =(1-v)^{1 / 2}\left(L^{p}-L^{p^{\prime}}-r^{2}\right) \delta^{-\gamma} \quad \text { with } \quad v \leq 1
\end{array}
$$

( $\mu>0$ actually, thanks to the hypothesis $\left|\rho \cdot \eta^{\prime}\right| \leq \mathrm{K} \delta^{-\gamma}$ ). Showing (86) amounts showing $v \geq 0$. With these new notations, inequality (89) is rewritten as

$$
\begin{aligned}
1+(\mathrm{C}-1) \mu \leq & 1-\frac{2}{L^{p-p^{\prime}-1}} \\
& +\frac{1}{L^{2\left(p-p^{\prime}-1\right)}}+\mathrm{C}\left(\frac{\mathrm{K}^{2}}{L^{2 p}}+\frac{2}{L^{p-p^{\prime}}}+\frac{2 r^{2}}{L^{p}}-\frac{1}{L^{2\left(p-p^{\prime}\right)}}-\frac{r^{4}}{L^{2 p}}-\frac{2 r^{2}}{L^{2 p-p^{\prime}}}\right) \\
& +\mathrm{C} v\left(1-\frac{L^{p^{\prime}}+r^{2}}{L^{p}}\right)^{2} .
\end{aligned}
$$

The left-hand side is larger or equal to 1 . But, when $L$ becomes large, the right-hand side is larger or equal to 1 only if $v>0$.

Lemma 5.4. Let $\left\{\rho_{1}, \ldots, \rho_{p}\right\}$ be a cluster around $x$, and let $\rho \in M_{r}$ be such that $\rho \notin \operatorname{span}\left\{\rho_{1}\right.$, $\left.\ldots, \rho_{p}\right\}$, but such that $\left\{\rho_{1}, \ldots, \rho_{p}, \rho\right\}$ is a cluster. If, given $\mathrm{K}<+\infty, L$ is taken large enough, then

$$
\eta \in \mathrm{B}\left(\rho_{1}, \ldots, \rho_{p}\right) \text { and }|\rho \cdot \eta| \leq \mathrm{K} \delta^{-\gamma} \quad \Rightarrow \quad \eta \in \mathrm{B}\left(\rho_{1}, \ldots, \rho_{p}, \rho\right) \text {. }
$$

Proof. Let us write $\rho=\rho_{p+1}$. Let $\eta \in \mathrm{B}\left(\rho_{1}, \ldots, \rho_{p}\right)$. By Lemma 5.2 and by hypothesis, it holds that

$$
\left|\eta-P\left(\eta, \pi\left(\rho_{1}\right) \cap \cdots \cap \pi\left(\rho_{p+1}\right)\right)\right|_{2} \leq \mathrm{C}\left(\mathrm{K} \delta^{-\gamma}+L^{p} \delta^{-\gamma}\right) \leq(L-1) L^{p} \delta^{-\gamma}
$$

if $L$ is large enough. Then

$$
\left|\eta-P\left(\eta, \pi\left(\rho_{1}\right) \cap \cdots \cap \pi\left(\rho_{p+1}\right)\right)\right|_{2} \leq L^{p+1} \delta^{-\gamma}
$$

and, for every $\rho_{1}^{\prime}, \ldots, \rho_{p^{\prime}}^{\prime} \in M_{r} \cap \operatorname{span}\left(\rho_{1}, \ldots, \rho_{p+1}\right)$, with $p^{\prime}<p+1$,

$$
\begin{aligned}
\left|P\left(\eta, \pi\left(\rho_{1}^{\prime}\right) \cap \cdots \cap \pi\left(\rho_{p \prime}^{\prime}\right)\right)-P\left(\eta, \pi\left(\rho_{1}\right) \cap \cdots \cap \pi\left(\rho_{p+1}\right)\right)\right|_{2} & \leq\left|\eta-P\left(\eta, \pi\left(\rho_{1}\right) \cap \cdots \cap \pi\left(\rho_{p+1}\right)\right)\right|_{2} \\
& \leq\left(L^{p+1}-L^{p}\right) \delta^{-\gamma} \leq\left(L^{p+1}-L^{p^{\prime}}\right) \delta^{-\gamma} .
\end{aligned}
$$

This shows $\eta \in \mathrm{B}\left(\rho_{1}, \ldots, \rho_{p}, \rho\right)$.

Proof of Proposition 5.1. Let us start with the first claim. Let $\rho \in M_{r}$ be such that $\operatorname{Supp}(\rho)$ $\subset \mathrm{B}(x, 4 r)$, and let $\eta \in \mathbb{R}^{N}$ be such that $\theta_{x}(\eta)>0$. On the one hand, from the definition (78) of $\theta_{x}$, it holds that there exists $\eta^{\prime} \in \mathbb{R}^{N}$, with $\max _{x}\left|\eta_{x}^{\prime}\right| \leq 2 \delta^{-\gamma}$, such that $\eta+\eta^{\prime} \notin \mathrm{R}(x)$. On the other hand, since $\operatorname{Supp}(\rho) \subset \mathrm{B}(x, 4 r)$, we conclude that $\{\rho\}$ alone is a cluster around $x$ so that, if $\eta^{\prime \prime} \in \mathbb{R}^{N}$ is such that

$$
\frac{\left|\rho \cdot \eta^{\prime \prime}\right|}{|\rho|_{2}}=\left|\eta^{\prime \prime}-P\left(\eta^{\prime \prime}, \pi(\rho)\right)\right| \leq L \delta^{-\gamma},
$$

then $\eta^{\prime \prime} \in \mathrm{R}(x)$. We thus conclude that $\left.\mathrm{|}\left(\eta+\eta^{\prime}\right) \cdot \rho|>| \rho\right|_{2} L \delta^{-\gamma}>L \delta^{-\gamma}$, and so that

$$
|\eta \cdot \rho|=\left|\left(\eta+\eta^{\prime}\right) \cdot \rho-\eta^{\prime} \cdot \rho\right|>L \delta^{-\gamma}-4 \delta^{-\gamma} r^{2} .
$$


It follows that

$$
\left|\Delta_{\rho} E(\eta)\right|=\left.|2 \eta \cdot \rho+| \rho\right|^{2} \mid>2 L \delta^{-\gamma}-8 \delta^{-\gamma} r^{2}-4 r^{4}>2 \delta^{-\gamma}
$$

if $L$ is large enough and $\delta$ is small enough. We conclude that $\zeta_{\rho}(\eta)=\xi_{\delta-\gamma}\left(\Delta_{\rho} E(\eta)\right)=0$.

Let us then show the second part of the proposition. Since, by (49) and (53), the Hamiltonian $\widetilde{h}$ takes the form

$$
\widetilde{h}=\sum_{\rho \in M_{r}} \hat{A}_{\rho} \hat{b}_{\rho} \hat{\zeta}_{\rho}
$$

we have

$$
\left(\operatorname{ad}_{\tilde{h}} \hat{\theta}_{x}\right) \hat{P}_{\eta}=\sum_{\rho \in M_{r}}\left[\hat{A}_{\rho} \hat{b}_{\rho} \hat{\zeta}_{\rho}, \hat{\theta}_{x}\right] \hat{P}_{\eta}=\sum_{\rho \in M_{r}} b_{\rho}(\eta) \zeta_{\rho}(\eta)\left(\Delta_{\rho} \theta_{x}(\eta)\right) \hat{A}_{\rho} \hat{P}_{\eta}
$$

It is thus enough to show that for all $\rho \in M_{r}$ and for all $\eta \notin \mathrm{S}_{n_{2}}(x)$ we have

$$
\zeta_{\rho}(\eta)=0 \quad \text { or } \quad \Delta_{\rho} \theta_{x}(\eta)=0 .
$$

Let us thus fix $\eta \in \mathbb{R}^{N}$ and $\rho \in M_{r}$. We will assume $\zeta_{\rho}(\eta) \neq 0$ and show that $\Delta_{\rho} \theta_{x}(\eta)=0$ follows. Note first that since

$$
\zeta_{\rho}(\eta)=\xi_{\delta-\gamma}\left(\Delta_{\rho} E(\eta)\right)=\xi_{\delta-\gamma}\left(2 \eta \cdot \rho+|\rho|^{2}\right)
$$

so $\zeta_{\rho}(\eta) \neq 0$ implies $|\eta \cdot \rho| \leq 2 \delta^{-\gamma}$ if $\delta$ is small enough. Now, by definition (78), we see that $\Delta_{\rho} \theta_{x}(\eta)$ $=0$ if, for every $\eta^{\prime} \in \mathbb{R}^{N}$ such that $\max _{x}\left|\eta_{x}^{\prime}\right| \leq 4 \delta^{-\gamma}$, it holds that

$$
\eta+\eta^{\prime} \in \mathrm{R}_{n_{2}}(x) \quad \Rightarrow \quad \eta+\eta^{\prime}+t \rho \in \mathrm{R}_{n_{2}}(x) \text { for all } t \text { such that }|t| \leq 2 r^{2} .
$$

Here, the maximal value allowed for $|t|$ is simply the maximal length of a move $\rho \in M_{r}$.

We distinguish three cases: In cases 1 and 2, it follows that $\Delta_{\rho} \theta_{x}(\eta)=0$ as required, and in case 3 , it follows that $\eta \in S_{n_{2}}(x)$ so that this case actually does not occur.

1. There exists a cluster $\left\{\rho_{1}, \ldots, \rho_{p}\right\}$ around $x$, with $p \leq n_{2}$, such that $\eta+\eta^{\prime} \in \mathrm{B}\left(\rho_{1}, \ldots, \rho_{p}\right)$ and that $\rho \perp \operatorname{span}\left\{\rho_{1}, \ldots, \rho_{p}\right\}$. It is then seen from the definition of $\mathrm{B}\left(\rho_{1}, \ldots, \rho_{p}\right)$ that, for every $t \in \mathbb{R}, \eta+\eta^{\prime}+t \rho \in \mathrm{B}\left(\rho_{1}, \ldots, \rho_{p}\right)$. Therefore $\eta+\eta^{\prime}+t \rho \in \mathrm{R}_{n_{2}}(x)$ for every $t \in \mathbb{R}$, and we are done.

2. There exists a cluster $\left\{\rho_{1}, \ldots, \rho_{p}\right\}$ around $x$, with $p \leq n_{2}$, such that $\eta+\eta^{\prime} \in \mathrm{B}\left(\rho_{1}, \ldots, \rho_{p}\right)$ and that $\rho \in \operatorname{span}\left\{\rho_{1}, \ldots, \rho_{p}\right\}$. Since $|\rho \cdot \eta| \leq 2 \delta^{-\gamma}$ and since $\max _{x}\left|\eta_{x}\right| \leq 4 \delta^{-\gamma}$, it holds that $\mid \rho \cdot(\eta$ $\left.+\eta^{\prime}\right) \mid \leq\left(8 r^{2}+2\right) \delta^{-\gamma}$. Then, by Lemma 5.3, for $|t| \leq \delta^{-\gamma}$ we still have $\eta+\eta^{\prime}+t \rho \in \mathrm{B}\left(\rho_{1}, \ldots\right.$, $\left.\rho_{p}\right)$ if $L$ was chosen large enough. So if we take $\delta$ small enough so that $\delta^{-\gamma} \geq 2 r^{2}$, we are done.

3. If neither case 1 nor case 2 is realized, then for any cluster $\left\{\rho_{1}, \ldots, \rho_{p}\right\}$ around $x$, with $p \leq n_{2}$ and such that $\eta+\eta^{\prime} \in \mathrm{B}\left(\rho_{1}, \ldots, \rho_{p}\right)$, it holds that $\rho \notin \operatorname{span}\left\{\rho_{1}, \ldots, \rho_{p}\right\}$, and that $\rho \not \mathcal{L} \operatorname{span}\left\{\rho_{1}\right.$, $\left.\ldots, \rho_{p}\right\}$. Let us see that, since we assume that $\eta \notin \mathrm{S}_{n_{2}}(x)$, this case actually does not happen. First, for all these clusters, we should have $p=n_{2}$. Indeed, otherwise $\left\{\rho_{1}, \ldots, \rho_{p}, \rho\right\}$ would form a cluster around $x$ containing $p+1 \leq n_{2}$ independent vectors. We would then conclude as in case 2 that $\left|\rho \cdot\left(\eta+\eta^{\prime}\right)\right| \leq\left(4 r^{2}+2\right) \delta$ so that, by Lemma $5.4, \eta+\eta^{\prime} \in \mathrm{B}\left(\rho_{1}, \ldots, \rho_{p}, \rho\right)$ if $L$ has been chosen large enough. This would contradict the assumption ensuring that we are in case 3. So $p=n_{2}$ should hold. Writing $\eta^{\prime \prime}=\eta+\eta^{\prime}$, we should then conclude from the definition of $\mathrm{B}\left(\rho_{1}, \ldots, \rho_{p}\right)$ that, for $1 \leq j \leq n_{2}$,

$$
\left|\rho_{j} \cdot \eta^{\prime \prime}\right|=\left|\rho_{j}\right|_{2}\left|\eta^{\prime \prime}-P\left(\eta^{\prime \prime}, \pi\left(\rho_{j}\right)\right)\right|_{2} \leq\left|\rho_{j}\right|_{2}\left|\eta^{\prime \prime}-P\left(\eta^{\prime \prime}, \pi\left(\rho_{1}\right) \cap \cdots \cap \pi\left(\rho_{n_{2}}\right)\right)\right|_{2} \leq\left|\rho_{j}\right|_{2} L^{n_{2}} \delta^{-\gamma} \text {. }
$$

But then

$$
\left|\rho_{j} \cdot \eta\right|=\left|\rho_{j} \cdot\left(\eta+\eta^{\prime}\right)-\rho_{j} \cdot \eta^{\prime}\right| \leq\left|\rho_{j} \cdot \eta^{\prime \prime}\right|+\left|\rho_{j} \cdot \eta^{\prime}\right| \leq\left|\rho_{j}\right|_{2} L^{n_{2}} \delta^{-\gamma}+4 r^{2} \delta^{-\gamma} \leq L^{n_{2}+1} \delta^{-\gamma}
$$

if $L$ is large enough. This would contradict $\eta \notin \mathrm{S}_{n_{2}}(x)$.

\section{PROOF OF THEOREM 2.3}

What remains to be done in order to prove Theorem 2.3 is to split the Hamiltonian in a left part and a right part. Our goal is to split the Hamiltonian such that there are no hopping terms in the Hamiltonian giving rise to transport across the cut. At the same time, we want to make our cut as 
close to a given site $a \in \mathbb{Z}_{N}$ as possible. But in Sec. V, we learned that the presence of hopping terms depends on the particle configuration. We see then that for some configurations, there will be no resonances around the chosen site $a$, and for such configurations we can split the Hamiltonian at $a$. For many other configurations, there are resonances around $a$, but there might be a spot nearby where there are no resonances, so we can still make our cut in the vicinity of the chosen site $a$. Finally, for a very sparse set $Z$ of configurations, there are no resonance free spots close enough to $a$. In that case, we do have transport across the cut, and the smallness comes from the small measure of $Z$ under the Gibbs state.

\section{A. New decomposition of the Hamiltonian}

The original splitting of the Hamiltonian in a left and right piece that was used in the definition of the current (15) through the bond $(a, a+1)$ corresponds for the reduced Hamiltonian to the following decomposition:

$$
\hat{h}=\hat{h}_{\leq a}^{\mathrm{O}}+\hat{h}_{>a}^{\mathrm{O}}:=\sum_{x \leq a} \hat{h}_{x}+\sum_{x>a} \hat{h}_{x},
$$

leading to a reduced current

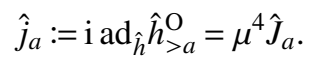

We will now obtain a new decomposition of the Hamiltonian that is equivalent to the one above from the point of view of the conductivity, but leading to an instantaneous current that vanishes for most of the configurations in the Gibbs state at small chemical potential.

Let $n_{3} \geq 1$. For $x \in \mathrm{B}\left(a, n_{3}\right)$, we define

$$
\begin{aligned}
& \vartheta_{a, x}=\frac{1}{\mathcal{N}}\left(\left(\prod_{y \in \mathrm{B}\left(a, n_{3}\right)} \theta_{y}\right) \delta_{a, x}+\left(1-\prod_{y \in \mathrm{B}\left(a, n_{3}\right)} \theta_{y}\right) \theta_{x}\right), \\
& \vartheta_{a, *}=\frac{1}{\mathcal{N}} \prod_{y \in \mathrm{B}\left(a, n_{3}\right)}\left(1-\theta_{y}\right) .
\end{aligned}
$$

with the normalization factor

$$
\mathcal{N}=\left(\prod_{y \in \mathrm{B}\left(a, n_{3}\right)} \theta_{y}\right)+\left(1-\prod_{y \in \mathrm{B}\left(a, n_{3}\right)} \theta_{y}\right)\left(\sum_{x \in \mathrm{B}\left(a, n_{3}\right)} \theta_{x}\right)+\prod_{y \in \mathrm{B}\left(a, n_{3}\right)}\left(1-\theta_{y}\right)
$$

chosen so that

$$
\sum_{x \in \mathrm{B}\left(a, n_{3}\right)} \vartheta_{a, x}+\vartheta_{a, *}=1
$$

and satisfying $\mathcal{N} \geq 1$. We then define

$$
\begin{aligned}
& \widetilde{h}_{\leq a}=\sum_{x \in \mathrm{B}\left(a, n_{3}\right)}\left(\sum_{y \leq x} \widetilde{h}_{y}\right) \hat{\vartheta}_{a, x}+\left(\sum_{y \leq a} \widetilde{h}_{y}\right) \hat{\vartheta}_{a, *}, \\
& \widetilde{h}_{>a}=\sum_{x \in \mathrm{B}\left(a, n_{3}\right)}\left(\sum_{y>x} \widetilde{h}_{y}\right) \hat{\vartheta}_{a, x}+\left(\sum_{y>a} \widetilde{h}_{y}\right) \hat{\vartheta}_{a, *} .
\end{aligned}
$$

It holds that

$$
\widetilde{h}=\widetilde{h}_{\leq a}+\widetilde{h}_{>a} .
$$

By the first point of Proposition 4.1, we finally define a new decomposition

$$
\hat{h}=\hat{h}_{\leq a}+\hat{h}_{>a}=\mathcal{T}_{n_{1}}\left(R \widetilde{\hat{h}}_{\leq a}\right)+\mathcal{T}_{n_{1}}\left(R \tilde{\hat{h}}_{>a}\right)
$$

\section{B. Definition of $\hat{u}_{a}$ and $\hat{g}_{a}$}

From the definitions (90), (91), and (97), and applying the second point of Proposition 4.1, we find that 


$$
\begin{aligned}
& \hat{j}_{a}=\mathrm{i} \operatorname{ad}_{\hat{h}} \hat{h}_{>a}^{\mathrm{O}}=\mathrm{i} \operatorname{ad}_{\hat{h}}\left(\hat{h}_{>a}^{\mathrm{O}}-\hat{h}_{>a}\right)+\mathrm{i} \operatorname{ad}_{\hat{h}} \hat{h}_{>a}
\end{aligned}
$$

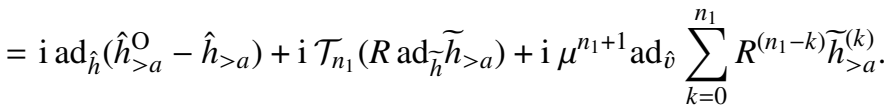

Let us call $n_{0}$ the number $n$ appearing in the statement of Theorem 2.3. We define symmetric operators

$$
\begin{aligned}
\hat{u}_{a} & =\hat{h}_{>a}^{\mathrm{O}}-\hat{h}_{>a}-\omega_{\mu}\left(\hat{h}_{>a}^{\mathrm{O}}-\hat{h}_{>a}\right), \\
\mu^{n_{0}+1} \hat{g}_{a} & =\mathrm{i} \mathcal{T}_{n_{1}}\left(R \operatorname{ad}_{\bar{h}} \widetilde{h}_{>a}\right)+\mathrm{i} \mu^{n_{1}+1} \operatorname{ad}_{\hat{v}} \sum_{k=0}^{n_{1}} R^{\left(n_{1}-k\right)} \widetilde{h}_{>a}^{(k)} .
\end{aligned}
$$

Then

$$
\hat{j}_{a}=\operatorname{iad}_{\hat{h}} \hat{u}_{a}+\mu^{n_{0}+1} \hat{g}_{a} .
$$

We notice that $\omega_{\mu}\left(\hat{g}_{a}\right)=0$ since $\mu^{n_{0}+1} \omega_{\mu}\left(\hat{g}_{a}\right)=\omega_{\mu}\left(\operatorname{ad}_{\hat{h}} \hat{h}_{>a}\right)=0$, by invariance of the Gibbs state.

\section{Locality}

Let us show that the operators $\hat{u}_{a}$ and $\hat{g}_{a}$ are local, meaning that their support consists of sites $z$ with $|z-a| \leq \mathrm{C}_{n_{0}}$, for some constant $\mathrm{C}_{n_{0}}<+\infty$. To study $\hat{u}_{a}$, we observe that

$$
\hat{h}_{>a}^{\mathrm{O}}-\hat{h}_{>a}=-\left(\hat{h}_{\leq a}^{\mathrm{O}}-\hat{h}_{\leq a}\right) .
$$

Let us see that $\hat{h}_{>a}^{\mathrm{O}}-\hat{h}_{>a}$ is supported on sites $z$ with $z \geq a-\left(n_{3}+\left(n_{2}+5\right) r\right)$. The operator $\hat{h}_{>a}^{\mathrm{O}}$ is supported on sites $z$ with $z \geq a$. To analyse $\hat{h}_{>a}$ defined by (97), we first notice that the functions $\vartheta_{a, x}$, with $x \in \mathrm{B}\left(a, n_{3}\right)$, and $\vartheta_{a, *}$, defined by (92-93), only depend on occupation numbers of sites $z$ with $z \geq a-\left(n_{3}+4 r+n_{2} r\right)$. By (96), the same holds true for $\widetilde{h}_{>a}$ since, for any $x \in \mathbb{Z}_{N}$, the operators $\widetilde{h}_{x}$ are supported on sites $z$ with $z \geq x-r$. By (53), we conclude that $R \widehat{h}_{>a}$, and so $\hat{h}_{>a}$, are supported on sites $z$ with $z \geq a-\left(n_{3}+6 r+n_{2} r\right)$. The same holds thus for $\hat{h}_{>a}^{\mathrm{O}}-\hat{h}_{>a}$. We can similarly show that $\hat{h}_{\leq a}^{\mathrm{O}}-\hat{h}_{\leq a}$ is supported on sites $z$ with $z \leq a+\left(n_{3}+\left(n_{2}+6\right) r\right)$. We conclude that $\hat{u}_{a}$ defined by (100) is supported on sites $z$ with $|z-a| \leq\left(n_{3}+\left(n_{2}+9\right) r\right)$.

We then readily conclude that $\hat{g}_{a}$ is local as well since, going back to (98), we see that $\mu^{n_{0}+1} g_{a}$ is the sum of two local functions,

$$
\mu^{n_{0}+1} \hat{g}_{a}=\mathrm{i} \mathrm{ad}_{\hat{h}} \hat{h}_{>a}=\hat{j}_{a}-\mathrm{i} \operatorname{ad}_{\hat{h}}\left(\hat{h}_{>a}^{\mathrm{O}}-\hat{h}_{>a}\right) .
$$

\section{An expression for $\operatorname{ad}_{\tilde{h}} \widetilde{h}_{>a}$}

We have

$$
\begin{aligned}
\operatorname{ad}_{\tilde{h}} \widetilde{h}_{>a}= & \sum_{x \in \mathrm{B}\left(a, n_{3}\right)}\left(\operatorname{ad}_{\tilde{h}} \sum_{y>x} \widetilde{h}_{y}\right) \hat{\vartheta}_{a, x}+\left(\operatorname{ad}_{\tilde{h}} \sum_{y>a} \widetilde{h}_{y}\right) \hat{\vartheta}_{a, *} \\
& +\sum_{x \in \mathrm{B}\left(a, n_{3}\right)}\left(\sum_{y>x} \widetilde{h}_{y}\right)\left(\operatorname{ad}_{\tilde{h}} \hat{\vartheta}_{a, x}\right)+\left(\sum_{y>a} \widetilde{h}_{y}\right)\left(\operatorname{ad}_{\tilde{h}} \hat{\vartheta}_{a, *}\right) .
\end{aligned}
$$

Let us show that the terms in the first sum in the right-hand side vanish, i.e.,

$$
\left(\operatorname{ad}_{\tilde{h}} \sum_{y>x} \widetilde{h}_{y}\right) \hat{\vartheta}_{a, x}=0 \quad \text { for all } \quad x \in \mathrm{B}\left(a, n_{3}\right)
$$

Thanks to the presence of the operator $\mathcal{R}$ in (49), and thanks to (53), we decompose $\widetilde{h}_{x}$ as

$$
\widetilde{h}_{x}=\hat{d}_{x}+\sum_{\substack{\rho \in M_{r} \backslash\{0\}: \\ \operatorname{Supp}(\rho) \subset \mathrm{B}(x, r)}} \hat{A}_{\rho} \hat{b}_{\rho} \hat{\zeta}_{\rho} .
$$

We also have

$$
\operatorname{ad}_{\tilde{h}} \sum_{y>x} \widetilde{h}_{y}=\left[\sum_{z \leq x} \widetilde{h}_{z}, \sum_{y>x} \widetilde{h}_{y}\right]=\sum_{\substack{z \leq x<y \\|z-y| \leq 2 r}}\left[\widetilde{h}_{z}, \widetilde{h}_{y}\right] .
$$


Fix now $\eta \in \Omega_{N}$. The claim (104) is certainly true if $\vartheta_{a, x}(\eta)=0$. We suppose that this is not the case and proceed to show that then

$$
\left(\operatorname{ad}_{\tilde{h}} \sum_{y>x} \widetilde{h}_{y}\right) \hat{P}_{\eta}=0
$$

This expression consists entirely of terms of the forms

$$
\left[\hat{d}_{z}, \hat{d}_{y}\right] \hat{P}_{\eta}, \quad\left[\hat{d}_{z}, \hat{A}_{\rho} \hat{b}_{\rho} \hat{\zeta}_{\rho}\right] \hat{P}_{\eta}, \quad\left[\hat{A}_{\rho} \hat{b}_{\rho} \hat{\zeta}_{\rho}, \hat{d}_{y}\right] \hat{P}_{\eta}, \quad \text { and } \quad\left[\hat{A}_{\rho} \hat{b}_{\rho} \hat{\zeta}_{\rho}, \hat{A}_{\rho^{\prime}} \hat{b}_{\rho^{\prime}} \hat{\zeta}_{\rho^{\prime}}\right] P_{\eta}
$$

with $\rho, \rho^{\prime} \in M_{r} \backslash\{0\}$ and $\operatorname{Supp}(\rho), \operatorname{Supp}\left(\rho^{\prime}\right) \subset \mathrm{B}(x, 4 r)$. The first of these vanishes because it is a commutator of diagonal operators. The others can all be rearranged so that a factor $\hat{\zeta}_{\rho} \hat{P}_{\eta}=\zeta_{\rho}(\eta) \hat{P}_{\eta}$ with $\rho \in M_{r} \backslash\{0\}$ and $\operatorname{Supp}(\rho) \subset \mathrm{B}(x, 4 r)$ appears. We will thus be done if we can show that $\zeta_{\rho}(\eta)$ $=0$ for all such $\rho$.

But we have $\vartheta_{a, x}(\eta)>0$ hence $\theta_{x}(\eta)>0$. It then follows from the first point of Proposition 5.1 that $\zeta_{\rho}(\eta)=0$ for all $\rho \in M_{r} \backslash\{0\}$ and $\operatorname{Supp}(\rho) \subset \mathrm{B}(x, 4 r)$ and so we are done.

The commutator (103) is now rewritten as

$$
\begin{aligned}
\operatorname{ad}_{\tilde{h}} \widetilde{h}_{>a}= & {\left[\sum_{a-r \leq x \leq a} \widetilde{h}_{x}, \sum_{a<y \leq a+r} \widetilde{h}_{y}\right] \hat{\vartheta}_{a, *} } \\
& +\sum_{x \in \mathrm{B}\left(a, n_{3}\right)}\left(\sum_{x<y \leq a+n_{3}} \widetilde{h}_{y}\right)\left(\operatorname{ad}_{\tilde{h}} \hat{\vartheta}_{a, x}\right)+\left(\sum_{a<y \leq a+n_{3}} \widetilde{h}_{y}\right)\left(\operatorname{ad}_{\tilde{h}} \hat{\vartheta}_{a, *}\right) .
\end{aligned}
$$

\section{E. Definition of an exceptional set $Z \subset \Omega$}

Let $\mathrm{Z} \subset \mathbb{R}^{N}$ be such that $\eta \in \mathrm{Z}$ if and only if there exists $n_{2}$ linearly independent vectors $\rho_{1}, \ldots, \rho_{n_{2}} \in M_{r}$ such that $\left(\cup_{j} \operatorname{Supp}\left(\rho_{j}\right)\right) \subset \mathrm{B}\left(a, 2 n_{3}\right)$ and such that $\left|\rho_{j} \cdot \eta\right| \leq L^{n_{2}+1} \delta^{-\gamma}$ for $1 \leq j$ $\leq n_{2}$.

We also consider for any $s>0$ a broadening of the set $Z$,

$$
\mathbf{Z}_{s}:=\left\{\eta \in \Omega_{N}: B(\eta, s) \cap \mathbf{Z} \neq \emptyset\right\} .
$$

Lemma 6.1. If, given $n_{1}$ and $n_{2}$, the numbers $L$ and $n_{3}$ have been taken large enough, then

1. If $a d_{\widetilde{h}} \widetilde{h}_{>a} \hat{P}_{\eta} \neq 0$, then $\eta \in \mathrm{Z}$.

2. There exists a constant $\mathrm{C}=\mathrm{C}\left(L, n_{1}, n_{2}, n_{3}, s\right)<+\infty$ such that $\omega_{\mu}\left(\hat{P}_{Z_{s}}\right) \leq \mathrm{C} \mu^{n_{2}} \delta^{-\gamma n_{2}}$.

Proof. Let us start with the first point. From (107), we conclude that, if $\left(\operatorname{ad}_{\tilde{h}} \widetilde{h}_{>a}\right) \hat{P}_{\eta} \neq 0$, then at

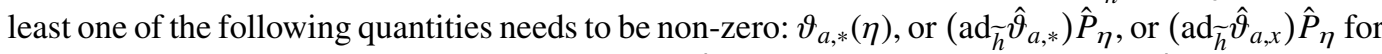
some $x \in \mathrm{B}\left(a, n_{3}\right)$. But if $\operatorname{ad}_{\tilde{h}}\left(\prod_{x \in \mathrm{B}\left(a, n_{3}\right)}\left(1-\hat{\theta}_{x}\right)\right) \hat{P}_{\eta} \neq 0$, then $\left(\prod_{x \in \mathrm{B}\left(a, n_{3}\right)}\left(1-\hat{\theta}_{x}\right)\right) \hat{P}_{\eta^{\prime}} \neq 0$ for some $\eta^{\prime} \in \Omega_{N}$ with $\left|\eta-\eta^{\prime}\right|_{2} \leq r$. Therefore, by inspection of the definitions (92) and (93), the condition $\left(\operatorname{ad}_{\widetilde{h}} \widetilde{h}_{>a}\right) \hat{P}_{\eta} \neq 0$ implies actually

$$
\left(\prod_{x \in \mathrm{B}\left(a, n_{3}\right)}\left(1-\hat{\theta}_{x}\right)\right) P_{\eta^{\prime}} \neq 0 \quad \text { for some } \quad \eta^{\prime} \in \Omega_{N} \quad \text { with } \quad\left|\eta-\eta^{\prime}\right|_{2} \leq r
$$

or

$$
\left(\operatorname{ad}_{\tilde{h}} \hat{\theta}_{x}\right) \hat{P}_{\eta} \neq 0 \quad \text { for some } x \in \mathrm{B}\left(a, n_{3}\right) .
$$

In the first case, $\theta_{x}\left(\eta^{\prime}\right)<1$ for all $x \in \mathrm{B}\left(a, n_{3}\right)$. But then, by definition (78), there exists some $\rho \in \mathbb{R}^{N}$ with $\max _{y}\left|\rho_{y}\right| \leq 2 \delta^{-\gamma}$ such that $\eta^{\prime \prime}=\eta^{\prime}+\rho \in \mathrm{R}(x)$. There exists therefore a cluster $\left\{\rho_{1}, \ldots\right.$, $\left.\rho_{p}\right\}$ around $x$, with $p \leq n_{2}$, such that (77) holds. This implies

$$
\left|\rho_{1} \cdot \eta^{\prime \prime}\right|=\left|\rho_{1}\right|_{2}\left|\eta^{\prime \prime}-P\left(\eta^{\prime \prime}, \pi\left(\rho_{1}\right)\right)\right|_{2} \leq\left|\rho_{1}\right|_{2}\left|\eta^{\prime \prime}-P\left(\eta^{\prime \prime}, \pi\left(\rho_{1}\right) \cap \cdots \cap \pi\left(\rho_{p}\right)\right)\right|_{2} \leq\left|\rho_{1}\right|_{2} L^{p} \delta^{-\gamma}
$$

and therefore $\left|\eta^{\prime} \cdot \rho_{1}\right| \leq\left|\rho_{1}\right|_{2} L^{p} \delta^{-\gamma}+r^{2} \delta^{-\gamma}$, and hence

$$
\left|\eta \cdot \rho_{1}\right|=\left|\eta^{\prime} \cdot \rho_{1}+\left(\eta-\eta^{\prime}\right) \cdot \rho_{1}\right| \leq\left|\rho_{1}\right|_{2} L^{p} \delta^{-\gamma}+r^{2} \delta^{-\gamma}+r \leq L^{n_{2}+1} \delta^{-\gamma}
$$

if $L$ is large enough, and using that $p \leq n_{2}$. 
It holds by definition of a cluster around $x$ that $\operatorname{Supp}\left(\rho_{1}\right) \subset \mathrm{B}(x, 4 r)$. Let us now take another $x^{\prime}$ such that $\theta_{x^{\prime}}\left(\eta^{\prime}\right)<1$ and $\left|x-x^{\prime}\right|>4 r$. Then the same reasoning gives a vector $\rho_{1}^{\prime} \neq \rho_{1}$ satisfying again $\left|\eta \cdot \rho_{1}^{\prime}\right| \leq L^{n+1} \delta^{-\gamma}$. By taking $n_{3}$ large enough, we can find $n_{2}$ linearly independent vectors and thus guarantee that $\eta \in \mathbf{Z}$.

Suppose now that $\operatorname{ad}_{\tilde{h}} \hat{\theta}_{x} \hat{P}_{\eta} \neq 0$ for some $x \in \mathrm{B}\left(a, n_{3}\right)$. It then follows from the second assertion of Proposition 5.1 that $\eta \in \mathrm{S}(x)$ so that, by definition, there exists a cluster $\left\{\rho_{1}, \ldots, \rho_{n_{2}}\right\}$ around $x$ such that $\left|\eta \cdot \rho_{j}\right| \leq L^{n_{2}+1} \delta^{-\gamma}$. This implies $\eta \in \mathbf{Z}$.

We now move to the second claim of the lemma. Since the Gibbs state factorises in the number basis, we find

$$
\begin{aligned}
\omega_{\mu}\left(\hat{P}_{Z_{s}}\right) & =\frac{\sum_{\eta \in \Omega_{N}} \chi \mathrm{Z}_{s}(\eta) \mathrm{e}^{-\mu|\eta|_{1}}}{\sum_{\eta \in \Omega_{N}} \mathrm{e}^{-\mu|\eta|_{1}}}=\frac{\sum_{\eta \in \mu \Omega_{N}} \chi \mathrm{Z}_{s}\left(\mu^{-1} \eta\right) \mathrm{e}^{-|\eta|_{1}}}{\sum_{\eta \in \mu \Omega_{N}} \mathrm{e}^{-|\eta|_{1}}} \\
& \leq C\left(n_{3}\right) \frac{\int \chi \mathrm{Z}_{s}\left(\mu^{-1} \eta\right) \prod_{x \in B\left(a, 2 n_{3}\right)} \mathrm{e}^{-\eta_{x}} \mathrm{~d} \eta_{x}}{\int \prod_{x \in B\left(a, 2 n_{3}\right)} \mathrm{e}^{-\eta_{x}} \mathrm{~d} \eta_{x}}
\end{aligned}
$$

for $\mu$ small enough. It then follows by exploiting that the set $Z_{s}$ is a finite union of cylinders whose bases have volume of order $\delta^{-\gamma n_{2}}$ that

$$
\omega_{\mu}\left(\hat{P}_{Z_{s}}\right) \leq C\left(\mu \delta^{-\gamma}\right)^{n_{2}}
$$

\section{F. Bounds on the norms of $\hat{u}_{a}$ and $\hat{g}_{a}$}

In this subsection, we fix $\delta=\mu$, so we are dealing again with the Bose-Hubbard Hamiltonian up to an overall factor of $\mu^{2}$. For $\hat{f} \in \mathcal{S}$, we again use the notation $\hat{f} \sim \delta^{-n}$ with the same meaning as in the proof of (54) and (55).

Let us first look at $\hat{u}_{a}$. Using (54) and (55) and the fact that the functions $\vartheta_{a, x}$ and $\vartheta_{a,{ }^{*}}$ are bounded, we find that the local function $\hat{h}_{>a}^{O}-\hat{h}_{>a}$ takes the form

$$
\begin{aligned}
\hat{h}_{>a}^{O}-\hat{h}_{>a} & =\sum_{n=0}^{n_{1}} \mu^{n}\left(\hat{h}_{>a}^{O}-\hat{h}_{>a}\right)^{(n)} \\
& =\hat{h}_{>a}^{O}-\mathcal{T}_{n_{1}}\left(R \widetilde{h}_{>a}\right)=\hat{h}_{>a}^{O}-\sum_{n=0}^{n_{1}} \mu^{n} \sum_{k=0}^{n} R^{(n-k)} \widetilde{h}_{>a}^{(k)} \sim \sum_{n=0}^{n_{1}} \mu^{n} \delta^{-2 n \gamma^{\prime}} .
\end{aligned}
$$

with the dominant contribution for each $n$ coming from the $k=0$ term.

But we can still improve on this for the $\left(\hat{h}_{>a}^{O}-\hat{h}_{>a}\right)^{(0)}$ term. Define $\mathrm{W} \subset \Omega$ as the set containing all $\eta$ such that $\theta_{x}(\eta)<1$ for some $x \in \mathrm{B}\left(a, n_{3}\right)$. By inspection of the definitions (92) and (93), we have

so writing

$$
\vartheta_{a, x}(\eta)=\delta_{a, x}, \quad \vartheta_{a, *}(\eta)=0, \quad \text { for } \eta \in \Omega \backslash \mathrm{W},
$$

$$
\begin{aligned}
\left(\hat{h}_{>a}^{O}-\hat{h}_{>a}\right)^{(0)} & =\sum_{x>a} \hat{d}_{x}-\widetilde{h}_{>a}^{(0)} \\
& =\sum_{x>a} \hat{d}_{x}-\sum_{x \in \mathrm{B}\left(a, n_{3}\right)}\left(\sum_{y>x} \hat{d}_{y}\right) \hat{\vartheta}_{a, x}-\left(\sum_{y>a} \hat{d}_{y}\right) \hat{\vartheta}_{a, *},
\end{aligned}
$$

we see that

$$
\left(\hat{h}_{>a}^{O}-\hat{h}_{>a}\right)^{(0)} \hat{P}_{\eta}=0, \quad \text { for } \eta \in \Omega \backslash \mathrm{W} .
$$

Therefore

$$
\omega_{\mu}\left(\left(\left(\hat{h}_{>a}^{O}-\hat{h}_{>a}\right)^{(0)}\right)^{2}\right)=\omega_{\mu}\left(\left(\left(\hat{h}_{>a}^{O}-\hat{h}_{>a}\right)^{(0)}\right)^{2} \hat{P}_{\mathrm{W}}\right) \leq \omega_{\mu}\left(\left(\left(\hat{h}_{>a}^{O}-\hat{h}_{>a}\right)^{(0)}\right)^{4}\right) \omega_{\mu}\left(\hat{P}_{\mathrm{W}}\right) .
$$

The operator $\left(\left(\hat{h}_{>a}^{O}-\hat{h}_{>a}\right)^{(0)}\right)^{4}$ is a finite polynomial of reduced field operators and diagonal operators that are uniformly bounded independent of $\mu$ and $\delta$. Its expectation value with respect to the Gibbs 
state can therefore be bounded by some constant. Concerning the projector $\hat{P}_{\mathrm{W}}$, we note that for any $\eta \in \mathrm{W}$ there is at least one $\rho \in M_{r}$ with $\operatorname{Supp}(\rho) \subset \mathrm{B}\left(a, n_{3}\right)$ and such that $|\eta \cdot \rho| \leq L^{n_{2}+1} \delta^{-\gamma}$ and thus $\omega\left(\hat{P}_{\mathrm{W}}\right) \leq C \mu \delta^{-\gamma}=\mu^{\gamma^{\prime}}$. Therefore

$$
\omega_{\mu}\left(\left(\left(h_{>a}^{O}-h_{>a}\right)^{(0)}\right)^{2} P \mathrm{~W}\right) \leq C \mu^{\gamma^{\prime}}
$$

for some constant $C$ depending on $n_{1}$ but, crucially, not depending on the volume thanks to the locality of $\left(h_{>a}^{O}-h_{>a}\right)^{(0)}$.

In a similar way, from (113) we obtain

$$
\omega_{\mu}\left(\left(\mu^{n}\left(\hat{h}_{>a}^{O}-\hat{h}_{>a}\right)^{(n)}\right)^{2}\right) \leq C \mu^{2 n} \delta^{-2 n \gamma^{\prime}}=C \mu^{2 n\left(1-\gamma^{\prime}\right)}
$$

for $1 \leq n \leq n_{1}$. Using [(115) and (116)] in (112) and since $\gamma^{\prime} \in(0,1 / 2)$, we therefore find

$$
\omega_{\mu}\left(\left(\hat{h}_{>a}^{O}-\hat{h}_{>a}\right)^{2}\right) \leq C \mu^{\gamma^{\prime}} .
$$

The constant $C$ depends on $n_{1}$ but not on the volume, and the bound holds for $\mu$ small enough. We conclude that $\omega_{\mu}\left(\hat{u}_{a}^{2}\right) \leq C \mu^{\gamma^{\prime}}$.

Next, we want $\omega_{\mu}\left(\hat{g}_{a}^{2}\right) \leq C \mu^{8}$ so that $\omega_{\mu}\left(\hat{G}_{a}^{2}\right)=\omega_{\mu}\left(\left(\mu^{-4} \hat{g}_{a}\right)^{2}\right)=\mu^{-8} \omega_{\mu}\left(\left(\hat{g}_{a}\right)^{2}\right)$ can be bounded by a constant. We start from the definition (101) and we note that both terms in this definition are local; for $\mathcal{T}_{n_{1}}\left(\operatorname{Rad}_{\widetilde{h}} \widetilde{h}_{>a}\right)$, this follows from the explicit expression in Sec. VI D and the other term is then local as a difference of local terms. We compute

$$
\omega_{\mu}\left(\hat{g}_{a}^{2}\right) \leq 2 \mu^{-2\left(n_{0}+1\right)} \omega_{\mu}\left(\left(\mathcal{T}_{n_{1}}\left(\operatorname{Rad}_{\tilde{h}} \widetilde{h}_{>a}\right)\right)^{2}\right)+2 \mu^{2\left(n_{1}-n_{0}\right)} \omega_{\mu}\left(\left(\operatorname{ad}_{\hat{v}} \sum_{k=0}^{n_{1}} R^{\left(n_{1}-k\right)} \widetilde{h}_{>a}^{(k)}\right)^{2}\right) .
$$

We look first at the second term and conclude by means of (54) and (55) that it is of the form

$$
\operatorname{ad}_{\hat{v}} \sum_{k=0}^{n_{1}} R^{\left(n_{1}-k\right)} \widetilde{h}_{>a}^{(k)} \sim \delta^{-2 n_{1} \gamma^{\prime}+\gamma} .
$$

We can thus bound, using locality, the fact that $\gamma^{\prime}<1 / 2$ and taking $\mu$ small enough,

$$
\mu^{2\left(n_{1}-n_{0}\right)} \omega_{\mu}\left(\left(\operatorname{ad}_{\hat{v}} \sum_{k=0}^{n_{1}} R^{\left(n_{1}-k\right)} \widetilde{h}_{>a}^{(k)}\right)^{2}\right) \leq C_{n_{0}} \mu^{8} \quad \text { if } \quad n_{1}=\left\lceil\frac{n_{0}+4-\gamma / 2}{1-2 \gamma^{\prime}}\right\rceil .
$$

We then analyse the first term in (118). By the first point of Lemma 6.1, the expression $\operatorname{ad}_{\bar{h}} \widetilde{h}_{>a} \hat{P}_{\eta}$ vanishes for all $\eta \in \Omega_{N} \backslash Z$, so remembering the definition (76) of the parameter $r$ we see that $\mathcal{T}_{n_{1}}\left(R \operatorname{ad}_{\widetilde{h}} \widetilde{h}_{>a}\right) \hat{P}_{\eta}$ certainly vanishes for all $\eta \in \Omega_{N} \backslash Z_{r}$ with

$$
\mathrm{Z}_{r}=\left\{\eta \in \Omega_{N}: \mathrm{B}(\eta, r) \cap \mathrm{Z} \neq \emptyset\right\},
$$

so

$$
\omega_{\mu}\left(\left(\mathcal{T}_{n_{1}}\left(R \operatorname{ad}_{\tilde{h}} \widetilde{h}_{>a}\right)\right)^{2}\right)=\omega_{\mu}\left(\left(\mathcal{T}_{n_{1}}\left(R \operatorname{ad}_{\tilde{h}} \widetilde{h}_{>a}\right)\right)^{2} \hat{P}_{Z_{r}}\right)
$$

Using (54) and (55) and $\gamma^{\prime}<1 / 2$, we find that

$$
\mathcal{T}_{n_{1}}\left(R \operatorname{ad}_{\tilde{h}} \widetilde{h}_{>a}\right)=\sum_{n=0}^{n_{1}} \mu^{n} \sum_{m=0}^{n} R^{(n-m)} \sum_{j=0}^{m}\left[\widetilde{h}^{(m-j)}, \widetilde{h}_{>a}^{(j)}\right] \sim \sum_{n=1}^{n_{1}} \mu^{n} \delta^{-2 n \gamma^{\prime}+4 \gamma^{\prime}+\gamma} \sim \mu^{1+2 \gamma^{\prime}+\gamma},
$$

where we have used the fact that $\left[\widetilde{h}^{(0)}, \widetilde{h}_{>a}^{(0)}\right]=0$ to start the last sum at $n=1$. We conclude that

$$
\omega_{\mu}\left(\left(\mathcal{T}_{n_{1}}\left(R \operatorname{ad}_{\tilde{h}} \widetilde{h}_{>a}\right)\right)^{4}\right) \leq C \mu^{4+8 \gamma^{\prime}+4 \gamma},
$$

and hence

$$
\omega_{\mu}\left(\left(\mathcal{T}_{n_{1}}\left(R \operatorname{ad}_{\tilde{h}} \widetilde{h}_{>a}\right)\right)^{2}\right) \leq \omega_{\mu}\left(\left(\mathcal{T}_{n_{1}}\left(R \operatorname{ad}_{\tilde{h}} \widetilde{h}_{>a}\right)\right)^{4}\right) \omega_{\mu}\left(\hat{P}_{Z_{r}}\right) \leq C \mu^{4(1+\gamma)+\left(8+n_{2}\right) \gamma^{\prime}}
$$

where we used the second point of Lemma 6.1. By taking $n_{2}=\left(2 n_{0}+6\right) / \gamma^{\prime}$, we conclude from (118) that $\omega_{\mu}\left(g_{a}^{2}\right) \leq C_{n_{0}} \mu^{8}$ if $\mu$ is small enough.

Thus, if we choose $\gamma^{\prime}=1 / 4$, then we have shown that

$$
\omega_{\mu}\left(u_{a}^{2}\right) \leq C \mu^{1 / 4} \quad \text { and } \quad \omega_{\mu}\left(g_{a}^{2}\right) \leq \mu^{8} .
$$




\section{G. Back to the original Bose-Hubbard Hamiltonian}

Defining the operators

$$
\hat{U}_{a}:=\mu^{-2} u_{a} \quad \text { and } \quad G_{a}:=\mu^{-4} g_{a},
$$

we decompose the current across the bond $(a, a+1)$ as

$$
\begin{aligned}
J_{a} & =\mathrm{iad}_{\hat{H}}\left(\hat{H}_{>a}\right)=\mathrm{iad}_{\mu^{-2} \hat{h}}\left(\mu^{-2} \hat{h}_{>a}^{O}\right)=\mu^{-4} \mathrm{iad}_{\hat{h}}\left(\hat{h}_{>a}^{O}\right) \\
& =\mu^{-4} \hat{j}_{a}=\mathrm{iad}_{\mu^{-2} \hat{h}}\left(\mu^{-2} \hat{u}_{a}\right)+\mu^{n_{0}+1}\left(\mu^{-4} \hat{g}_{a}\right)=\mathrm{i} \mathrm{ad}_{\hat{H}} \hat{U}_{a}+\mu^{n_{0}+1} \hat{G}_{a} .
\end{aligned}
$$

Furthermore, we have showed in Subsection VI F that

$$
\omega_{\mu}\left(\hat{U}_{a}^{2}\right)=\mu^{-4} \omega_{\mu}\left(\hat{u}_{a}^{2}\right) \leq C \mu^{-4} \mu^{1 / 4}<C \mu^{-4}
$$

and

$$
\omega_{\mu}\left(\hat{G}_{a}^{2}\right)=\mu^{-8} \omega_{\mu}\left(\hat{g}_{a}^{2}\right) \leq C_{n_{0}} .
$$

Taking $n=n_{0}$ concludes the Proof of Theorem 2.3.

\section{PROOF OF THEOREM 2.2}

Let $I=\left\{a_{1}+1, \ldots, a_{2}\right\} \subset \mathbb{Z}_{N}$ be an interval on the chain and let $\hat{H}_{I}=\sum_{x \in I} \hat{H}_{x}$ be the energy in the interval $I$. Since $\hat{H}_{I}=\hat{H}_{>a_{1}}-\hat{H}_{>a_{2}}$, we have

$$
\operatorname{iad}_{\hat{H}}\left(\hat{H}_{I}\right)=\operatorname{iad}_{\hat{H}}\left(\hat{H}_{>a_{1}}\right)-\mathrm{iad}_{\hat{H}}\left(\hat{H}_{>a_{2}}\right)=\hat{J}_{a_{1}}-\hat{J}_{a_{2}} .
$$

Up till this point, the arguments basically involved only algebra and no analysis, and we could understand operators as elements of the $*$-algebra $\mathcal{A}$. From this point onwards, we consider timeevolved operators and therefore we need to understand them as elements of the Hilbert space $\mathcal{B}$; see Lemma 2.1. Let us write $\hat{H}_{I}(t)$ for the energy in the interval $I$ at time $t$ in the Heisenberg picture. By Lemma 2.1, we get

$$
\hat{H}_{I}(t)-\hat{H}_{I}(0)=\mathrm{i} \int_{0}^{t} \mathrm{~d} s \operatorname{ad}_{\hat{H}}\left(\hat{H}_{I}\right)(s)=\int_{0}^{t} \mathrm{~d} s\left(\hat{J}_{a_{1}}(s)-\hat{J}_{a_{2}}(s)\right) .
$$

Using Theorem 2.3 and again Lemma 2.1, we find for any $a \in \mathbb{Z}_{N}$ that

$$
\int_{0}^{t} \mathrm{~d} s \hat{J}_{a}(s)=\int_{0}^{t} \mathrm{~d} s\left(\operatorname{iad}_{\hat{H}}\left(\hat{U}_{a}\right)(s)+\mu^{n+1} \hat{G}_{a}(s)\right)=\hat{U}_{a}(t)-\hat{U}_{a}(0)+\mu^{n+1} \int_{0}^{t} \mathrm{~d} s \hat{G}_{a}(s),
$$

so

$$
\hat{H}_{I}(t)-\hat{H}_{I}(0)=\sum_{j \in\{1,2\}}(-1)^{j}\left(\hat{U}_{a_{j}}(t)-\hat{U}_{a_{j}}(0)+\mu^{n+1} \int_{0}^{t} \mathrm{~d} s \hat{G}_{a_{j}}(s)\right) .
$$

It follows that

$$
\begin{aligned}
\omega_{\mu}\left(\left(\hat{H}_{I}(t)-\hat{H}_{I}(0)\right)^{2}\right) \leq 2 \omega_{\mu} & \left(\left(\sum_{j \in\{1,2\}}(-1)^{j}\left(\hat{U}_{a_{j}}(t)-\hat{U}_{a_{j}}(0)\right)\right)^{2}\right) \\
+ & 2 \mu^{2 n+2} \omega_{\mu}\left(\left(\sum_{j \in\{1,2\}}(-1)^{j} \int_{0}^{t} \mathrm{~d} s \hat{G}_{a_{j}}(s)\right)^{2}\right) .
\end{aligned}
$$

Using Cauchy-Schwarz, time-translation invariance of the Gibbs state and the bound of Theorem 2.3, the first term in this expression is bounded by

$$
\begin{gathered}
\sum_{j, k \in\{1,2\}}(-1)^{j+k}\left(\omega_{\mu}\left(\hat{U}_{a_{j}}(t) \hat{U}_{a_{k}}(t)\right)-\omega_{\mu}\left(\hat{U}_{a_{j}}(t) \hat{U}_{a_{k}}(0)\right)-\omega_{\mu}\left(\hat{U}_{a_{j}}(0) \hat{U}_{a_{k}}(t)\right)+\omega_{\mu}\left(\hat{U}_{a_{j}}(0) \hat{U}_{a_{k}}(0)\right)\right) \\
\leq 4 \sum_{j, k \in\{1,2\}}\left(\omega_{\mu}\left(\hat{U}_{a_{j}}^{2}\right) \omega_{\mu}\left(\hat{U}_{a_{k}}^{2}\right)\right)^{1 / 2} \leq C \mu^{-4} .
\end{gathered}
$$


Taking $0 \leq t \leq \mu^{-n}$, we can bound the second term in the same way by

$$
\begin{aligned}
4 \mu^{2 n+2} \sum_{j \in\{1,2\}} \omega_{\mu}\left(\left(\int_{0}^{t} \mathrm{~d} s \hat{G}_{a_{j}}(s)\right)^{2}\right) & =4 \mu^{2 n+2} \sum_{j \in\{1,2\}} \int_{0}^{t} \mathrm{~d} s \int_{0}^{t} \mathrm{~d} s^{\prime} \omega_{\mu}\left(\hat{G}_{a_{j}}(s) \hat{G}_{a_{j}}\left(s^{\prime}\right)\right) \\
& \leq 4 \mu^{2 n+2} \sum_{j \in\{1,2\}} \mu^{-2 n} \omega_{\mu}\left(\hat{G}_{a_{j}}^{2}\right) \leq C \mu^{2},
\end{aligned}
$$

thus proving the theorem.

\section{ACKNOWLEDGMENTS}

The authors benefit from funding by the InterUniversity Attraction Pole DYGEST (Belspo, Phase VII/18), from the DFG (German Research Fund) grant number RO 4522/1-1, and from the FWO (Flemish Research Fund) grant number G076216N.

${ }^{1}$ Abdul-Rahman, H., Nachtergaele, B., and Sims, R., "Localization properties of the disordered XY spin chain: A review of mathematical results with an eye toward many-body localization," Stolz, G., Ann. der Phys. 529, 1600280 (2017).

${ }^{2}$ Basko, D. M., Aleiner, I. L., and Altshuler, B. L., "Metal-insulator transition in a weakly interacting many-electron system with localized single-particle states," Ann. Phys. 321(5), 1126-1205 (2006).

${ }^{3}$ Benedikter, N., Porta, M., and Schlein, B., "Mean-field regime for bosonic systems," in Effective Evolution Equations from Quantum Dynamics (Springer, 2016), pp. 7-16

${ }^{4}$ Bera, S., De Tomasi, G., Weiner, F., and Evers, F., "Density propagator for many-body localization: Finite size effects, transient subdiffusion, and exponential decay,” Phys. Rev. Lett. 118, 196801 (2017).

${ }^{5}$ Carleo, G., Becca, F., Schiró, M., and Fabrizio, M., "Localization and glassy dynamics of many-body quantum systems," Sci. Rep. 2, 243 (2012).

${ }^{6}$ De Roeck, W. and Huveneers, F., "Asymptotic quantum many-body localization from thermal disorder," Commun. Math. Phys. 332(3), 1017-1082 (2014).

${ }^{7}$ De Roeck, W. and Huveneers, F., "Scenario for delocalization in translation-invariant systems," Phys. Rev. B 90(16), 165137 (2014).

${ }^{8}$ De Roeck, W. and Huveneers, F., "Asymptotic localization of energy in nondisordered oscillator chains," Commun. Pure Appl. Math. 68(9), 1532-1568 (2015).

${ }^{9}$ De Roeck, W., Huveneers, F., Müller, M., and Schiulaz, M., “Absence of many-body mobility edges,” Phys. Rev. B 93(1), 014203 (2016).

${ }^{10}$ Deutsch, J. M., "Quantum statistical mechanics in a closed system," Phys. Rev. A 43(4), 2046 (1991).

${ }^{11}$ Eisert, J., Friesdorf, M., and Gogolin, C., "Quantum many-body systems out of equilibrium," Nat. Phys. 11(2), 124-130 (2015).

${ }^{12}$ Gornyi, I. V., Mirlin, A. D., and Polyakov, D. G., "Interacting electrons in disordered wires: Anderson localization and low-t transport," Phys. Rev. Lett. 95(20), 206603 (2005).

${ }^{13}$ Grover, T. and Fisher, M. P. A., "Quantum disentangled liquids,” J. Stat. Mech.: Theory Exp. 2014(10), P10010.

${ }^{14}$ He, R.-Q. and Weng, Z.-Y., "On the possibility of many-body localization in a doped mott insulator," Sci. Rep. 6, 35208 (2016).

15 Hepp, K., “The classical limit for quantum mechanical correlation functions,” Commun. Math. Phys. 35(4), 265-277 (1974).

${ }^{16}$ Hickey, J. M., Genway, S., and Garrahan, J. P., "Signatures of many-body localisation in a system without disorder and the relation to a glass transition," J. Stat. Mech.: Theory Exp. 2016(5), 054047.

${ }^{17}$ Huse, D. A., Nandkishore, R., and Oganesyan, V., "Phenomenology of fully many-body-localized systems," Phys. Rev. B 90(17), 174202 (2014).

${ }^{18}$ Huveneers, F., "Drastic fall-off of the thermal conductivity for disordered lattices in the limit of weak anharmonic interactions," Nonlinearity 26(3), 837 (2013).

${ }^{19}$ Imbrie, J. Z., "On many-body localization for quantum spin chains," J. Stat. Phys. 163(5), 998-1048 (2016).

${ }^{20}$ Imbrie, J. Z., Ros, V., and Scardicchio, A., "Local integrals of motion in many-body localized systems," Ann. der Phys. 529, 1600278 (2017).

${ }^{21}$ Kagan, Y. and Maksimov, L. A., "Localization in a system of interacting particles diffusing in a regular crystal," Zhurnal Eksperimental'noi i Teoreticheskoi Fiziki 87, 348-365 (1984).

${ }^{22}$ Khemani, V., Pollmann, F., and Sondhi, S. L., "Obtaining highly excited eigenstates of many-body localized Hamiltonians by the density matrix renormalization group approach," Phys. Rev. Lett. 116(24), 247204 (2016).

${ }^{23}$ Kim, I. H. and Haah, J., "Localization from superselection rules in translationally invariant systems," Phys. Rev. Lett. 116(2), 027202 (2016).

${ }^{24}$ Kjäll, J. A., Bardarson, J. H., and Pollmann, F., "Many-body localization in a disordered quantum ising chain,” Phys. Rev. Lett. 113(10), 107204 (2014).

${ }^{25}$ Luitz, D. J., Laflorencie, N., and Alet, F., "Many-body localization edge in the random-field heisenberg chain," Phys. Rev. B 91(8), 081103 (2015).

${ }^{26}$ Mastropietro, V., "Localization in the ground state of an interacting quasi-periodic fermionic chain," Commun. Math. Phys. 342(1), 217-250 (2016).

${ }^{27}$ Nandkishore, R. and Huse, D. A., "Many body localization and thermalization in quantum statistical mechanics," Annu. Rev. Condens. Matter Phys. 6, 15-38 (2015). 
${ }^{28}$ Oganesyan, V. and Huse, D. A., "Localization of interacting fermions at high temperature," Phys. Rev. B 75(15), 155111 (2007).

${ }^{29} \mathrm{Pal}$, A. and Huse, D. A., "Many-body localization phase transition,” Phys. Rev. B 82(17), 174411 (2010).

${ }^{30}$ Papić, Z., Miles Stoudenmire, E., and Abanin, D. A., "Many-body localization in disorder-free systems: The importance of finite-size constraints," Ann. Phys. 362, 714-725 (2015).

${ }^{31}$ Pino, M., Ioffe, L. B., and Altshuler, B. L., "Nonergodic metallic and insulating phases of josephson junction chains," Proc. Natl. Acad. Sci. U. S. A. 113(3), 536-541 (2016).

${ }^{32}$ Pöschel, J., "Nekhoroshev estimates for quasi-convex Hamiltonian systems," Math. Z. 213(1), 187-216 (1993).

${ }^{33}$ Potter, A. C., Vasseur, R., and Parameswaran, S. A., "Universal properties of many-body delocalization transitions," Phys. Rev. X 5(3), 031033 (2015).

${ }^{34}$ Ros, V., Müller, M., and Scardicchio, A., "Integrals of motion in the many-body localized phase," Nucl. Phys. B 891, 420-465 (2015).

${ }^{35}$ Schiulaz, M. and Müller, M., "Ideal quantum glass transitions: Many-body localization without quenched disorder," AIP Conf. Proc. 1610, 11 (2014).

${ }^{36}$ Schiulaz, M., Silva, A., and Müller, M., "Dynamics in many-body localized quantum systems without disorder," Phys. Rev. B 91(18), 184202 (2015).

${ }^{37}$ Seiringer, R. and Warzel, S., "Decay of correlations and absence of superfluidity in the disordered Tonks-Girardeau gas," New J. Phys. 18(3), 035002 (2016).

${ }^{38}$ Serbyn, M., Papić, Z., and Abanin, D. A., "Local conservation laws and the structure of the many-body localized states," Phys. Rev. Lett. 111(12), 127201 (2013).

${ }^{39}$ Shapir, Y., Aharony, A., and Brooks Harris, A., "Localization and quantum percolation," Phys. Rev. Lett. 49(7), 486 (1982)

${ }^{40}$ Singh, R. and Shimshoni, E., "Localization due to interaction-enhanced disorder in bosonic systems," Ann. der Phys. 529, 1600309 (2017).

${ }^{41}$ Srednicki, M., "Chaos and quantum thermalization,” Phys. Rev. E 50(2), 888 (1994)

42 Stolz, G., "Many-body localization for disordered bosons," New J. Phys. 18(3), 031002 (2016).

${ }^{43}$ van Horssen, M., Levi, E., and Garrahan, J. P., "Dynamics of many-body localization in a translation-invariant quantum glass model," Phys. Rev. B 92(10), 100305 (2015).

44 Veselić, I., "Spectral analysis of percolation Hamiltonians," Math. Ann. 331(4), 841-865 (2005).

${ }^{45}$ Vosk, R., Huse, D. A., and Altman, E., "Theory of the many-body localization transition in one-dimensional systems," Phys. Rev. X 5(3), 031032 (2015).

${ }^{46}$ Yao, N. Y., Laumann, C. R., Cirac, J. I., Lukin, M. D., and Moore, J. E., "Quasi-many-body localization in translationinvariant systems," Phys. Rev. Lett. 117, 240601 (2016).

47 Žnidarič, M., Prosen, T., and Prelovšek, P., "Many-body localization in the heisenberg x x z magnet in a random field," Phys. Rev. B 77(6), 064426 (2008).

${ }^{48}$ The terminology is chosen for simplicity but it is unconventional. Given temperature $T$ and chemical potential $\tilde{\mu}$, the density matrix is $\propto \mathrm{e}^{-\frac{1}{T}(H-\tilde{\mu} \hat{N})}$. We consider the limit $T, \tilde{\mu} \rightarrow \infty$ such that $\mu:=-\tilde{\mu} / T$ remains finite. 